\title{
Fitting hyperelastic models to experimental data
}

\author{
R. W. Ogden, G. Saccomandi, I. Sgura
}

\begin{abstract}
This paper is concerned with determining material parameters in incompressible isotropic elastic strain-energy functions on the basis of a non-linear least squares optimization method by fitting data from the classical experiments of Treloar and Jones and Treloar on natural rubber. We consider three separate forms of strain-energy function, based respectively on use of the principal stretches, the usual principal invariants of the Cauchy-Green deformation tensor and a certain set of 'orthogonal' invariants of the logarithmic strain tensor. We highlight, in particular, (a) the relative errors generated in the fitting process and (b) the occurrence of multiple sets of optimal material parameters for the same data sets. This multiplicity can lead to very different numerical solutions for a given boundary-value problem, and this is illustrated for a simple example.
\end{abstract}

Keywords Constitutive laws, Elasticity, Elastomers, Finite deformation, Isotropic

1

\section{Introduction}

In this paper, we carry out a systematic analysis of the fitting of incompressible isotropic hyperelastic constitutive laws to experimental data, the determination of material parameters and the corresponding relative errors. For this purpose we use the original data of Treloar [1] and Jones and Treloar [2] for natural rubber, a non-linear least

\author{
R. W. Ogden \\ Department of Mathematics, \\ University of Glasgow, \\ Glasgow G12 8QW, Scotland, UK \\ e-mail: rwo@maths.gla.ac.uk \\ G. Saccomandi \\ Dipartimento di Ingegneria dell'Innovazione, \\ Sezione di Ingegneria Industriale, \\ Università degli Studi di Lecce, 73100 Lecce, Italy \\ e-mail: giuseppe.saccomandi@unile.it

\section{Sgura} \\ Dipartimento di Matematica, \\ Università degli Studi di Lecce, 73100 Lecce, Italy \\ e-mail: ivonne.sgura@unile.it
}

The work of G. S. was partially supported by GNFM, INDAM, Italy. The work of I. S. was partially supported by the Progetto Giovani Ricercatori Università di Lecce - MIUR 2001/2002. squares optimization tool from Matlab, and three specific material models due to Ogden [3], based on stretches, Pucci and Saccomandi [4], based on the usual invariants $I_{1}$ and $I_{2}$ of the Cauchy-Green deformation tensor, and Criscione et al. [5], based on a set of 'orthogonal' invariants of the logarithmic strain tensor.

An important aim of the paper is to show, by means of numerical examples, that the fitting of experimental data within the framework of the mechanics (or thermomechanics) of elastomeric solids is a very delicate question. In particular, non-uniqueness of 'optimal' material parameters often occurs, and this non-uniqueness may be reflected very markedly in the solution of boundary-value problems. The difficulties arising in the fitting procedure are intrinsic to the considered problem and are not in general dependent on the specific choice of constitutive law, although in some cases uniqueness may be achieved; one case in point is where the material constants occur linearly in the stress-deformation equations; another that of the Pucci-Saccomandi model discussed in Sect. 5. Moreover, the difficulties are not functions of the particular formulation, whether in terms of stretches or invariants. If, for example, polynomial models are used (with the material constants appearing linearly) then to obtain 'good' fits to the data for an extensive range of deformations it is necessary to employ high-order polynomials, which typically introduce numerical instabilities. On the other hand, if the material constants feature in a nonlinear way then non-uniqueness of the optimal set of constants can be the rule unless some intrinsic property of the problem is used to avoid this.

We begin, in Sect. 2, by summarizing the relevant equations that describe the stresses in a homogeneous pure strain of an incompressible isotropic elastic solid as derivatives of the strain-energy function and the important special cases of simple tension and equibiaxial tension. In Sect. 3 the optimization method to be used in the subsequent sections is described.

The Ogden model, with either six or eight material parameters, is the focus of attention in Sect. 4. It is shown that by fitting the Treloar data for simple tension (equibiaxial tension) the prediction for equibiaxial tension (simple tension) is not good. This is not surprising. On the other hand, if both sets of data are fitted together then good overall fits are obtained with reasonably low relative errors. Corresponding fits are performed for the full set of the Jones-Treloar biaxial data. An important feature of the results is that multiple sets of optimal values of the material parameters are generated by taking different 
starting values and/or by setting different levels of accuracy in the optimization procedure. This clearly has implications for the solution of boundary-value problems, and we emphasize this point by considering, in Sect. 7, a simple boundary-value problem in which significantly different numerical predictions of the solution are obtained by using different optimal sets of parameters. It also has serious implications in respect of implementation of material models in commercial software, particularly since non-uniqueness is inherent in problems of non-linear elasticity. Thus, careful mathematical analysis is needed to ensure confidence in the resulting numerical solutions.

In Sect. 5, for the Pucci and Saccomandi model, an extension of the Gent model [16] having three material parameters, we find that there is no multiplicity of optimal sets of parameters. This model fits the Treloar data well and also the Jones-Treloar data except in the compression range. We also report, in Sect. 7, the predictions of the different parameter sets obtained for this model for different sets of data in respect of the boundary-value problem mentioned above.

In Sect. 6, we examine the Criscione et al. [5] model on the same basis as for the other two models. We find that whilst this model gives a good fit to the Jones-Treloar data for moderate stretches with just five material parameters it does not fit the Treloar simple tension data well for higher stretches unless a very much larger number of parameters is used.

\section{2}

\section{Basic equations}

In this section we summarize briefly the equations of incompressible isotropic non-linear elasticity that are required for comparing the theory with experimental data in standard experimental protocols. The data that we shall consider in this paper relate to experiments in which the deformation is homogeneous. More specifically, we consider homogeneous deformations that can be classified as pure homogeneous strain, i.e. deformations of the form

$x_{1}=\lambda_{1} X_{1}, \quad x_{2}=\lambda_{2} X_{2}, \quad x_{3}=\lambda_{3} X_{3}$,

where $\left(X_{1}, X_{2}, X_{3}\right)$ are rectangular Cartesian coordinates that identify material particles in some unstressed reference configuration, $\left(x_{1}, x_{2}, x_{3}\right)$ are the corresponding coordinates after deformation with respect to the same axes, and the coefficients $\lambda_{1}, \lambda_{2}, \lambda_{3}$ are positive constants, referred to as the principal stretches of the deformation. The principal axes of the deformation coincide with the Cartesian coordinate directions and are fixed as the values of the stretches change.

In the theory of hyperelasticity there exists a strainenergy function (or stored-energy function), defined per unit volume and denoted $W$. It depends symmetrically on $\lambda_{1}, \lambda_{2}, \lambda_{3}$, i.e.

$W\left(\lambda_{1}, \lambda_{2}, \lambda_{3}\right)=W\left(\lambda_{1}, \lambda_{3}, \lambda_{2}\right)=W\left(\lambda_{2}, \lambda_{1}, \lambda_{3}\right)$,

and for an incompressible material the principal stretches satisfy the constraint

$\lambda_{1} \lambda_{2} \lambda_{3}=1$.

The principal Cauchy stresses $\sigma_{i}, i \in\{1,2,3\}$ (defined per unit cross-sectional area normal to the $x_{i}$ axis in the deformed configuration), are related to the stretches through $W$ according to the equations

$\sigma_{i}=\lambda_{i} \frac{\partial W}{\partial \lambda_{i}}-p$

where $p$ is a Lagrange multiplier associated with the constraint (3). Note that in (4) there is no summation over the repeated index $i$. The corresponding Biot (or nominal) stresses $t_{i}$ (defined per unit reference cross-sectional area) are the stresses that are often measured directly in experiments and are given by

$t_{i}=\frac{\partial W}{\partial \lambda_{i}}-p \lambda_{i}^{-1} \equiv \sigma_{i} \lambda_{i}^{-1}$.

Since the material considered is incompressible, only two stretches can be varied independently and hence biaxial tests are sufficient to determine the form of $W$. For this reason it has been found convenient to use the incompressibility constraint (3) to express the strain energy as a function of two independent stretches, and here we use $\lambda_{1}$ and $\lambda_{2}$ and the notation defined by

$\hat{W}\left(\lambda_{1}, \lambda_{2}\right)=W\left(\lambda_{1}, \lambda_{2}, \lambda_{1}^{-1} \lambda_{2}^{-1}\right)$.

This enables $p$ to be eliminated from (4) and leads to

$\sigma_{1}-\sigma_{3}=\lambda_{1} \frac{\partial \hat{W}}{\partial \lambda_{1}}, \quad \sigma_{2}-\sigma_{3}=\lambda_{2} \frac{\partial \hat{W}}{\partial \lambda_{2}}$

For the energy and stresses to vanish in the chosen reference configuration and for consistency with the classical theory of incompressible isotropic elasticity we must have

$\hat{W}(1,1)=0, \quad \hat{W}_{1}(1,1)=\hat{W}_{2}(1,1)=0$,

$\hat{W}_{12}(1,1)=2 \mu, \quad \hat{W}_{11}(1,1)=\hat{W}_{22}(1,1)=4 \mu$,

where the subscripts 1 and 2 signify differentiation with respect to $\lambda_{1}$ and $\lambda_{2}$, respectively, and $\mu(>0)$ is the shear modulus of the material in the reference configuration.

If the deformation (1) is applied to a thin sheet of material (a common situation experimentally) then a plane stress condition applies and we may set $\sigma_{3}=0$, the stress normal to the plane of the sheet. In terms of the nominal stresses we then have simply

$t_{1}=\frac{\partial \hat{W}}{\partial \lambda_{1}}, \quad t_{2}=\frac{\partial \hat{W}}{\partial \lambda_{2}}$,

which provides two equations relating $\lambda_{1}, \lambda_{2}$ and $t_{1}, t_{2}$ and therefore a simple basis for characterizing $\hat{W}$ from measured (homogeneous) biaxial data.

There are several special cases of the biaxial test which are important from the experimental point of view. These are simple tension and equibiaxial tension, which we now discuss separately and briefly, and pure shear, which we do not examine here. A subscript $\mathrm{s}$ or e will be used to label quantities associated with, respectively, simple tension and equibiaxial tension.

\section{(i) Simple tension}

For simple tension we set $t_{2}=0$ and $t_{1}=t$. Then, by symmetry, the incompressibility constraint yields 
$\lambda_{2}=\lambda_{3}=\lambda_{1}^{-1 / 2}$ and we write $\lambda_{1}=\lambda$. The strain energy may now be treated as a function of just $\lambda$, and we define $W_{s}(\lambda)=\hat{W}\left(\lambda, \lambda^{-\frac{1}{2}}\right)$,

and (9) reduces to

$t=W_{\mathrm{s}}^{\prime}(\lambda)$

where the prime indicates differentiation with respect to $\lambda$.

(ii) Equibiaxial tension

For equibiaxial tension we have $\lambda_{1}=\lambda_{2}$ (= $\lambda$, say), $\lambda_{3}=\lambda_{1}^{-2}$ and $t_{1}=t_{2}(=t$, say). Then, we define

$W_{\mathrm{e}}(\lambda)=\hat{W}(\lambda, \lambda)$,

and (9) reduces to

$t=\frac{1}{2} W_{\mathrm{e}}^{\prime}(\lambda)$.

For discussion of the possible ranges of values of the stretches in various tests we refer to Ogden [6].

In Sect. 4 we shall focus on fitting members of a specific class of energy functions to various sets of experimental data. The considered strain-energy functions have the form

$$
W=\sum_{i=1}^{\infty} \frac{\mu_{i}}{\alpha_{i}}\left(\lambda_{1}^{\alpha_{i}}+\lambda_{2}^{\alpha_{i}}+\lambda_{3}^{\alpha_{i}}-3\right),
$$

where each $\mu_{i}$ and $\alpha_{i}$ is a material constant, the latter not necessarily being integers (Ogden $[3,7,8]$ ). For practical purposes the sum in (14) is restricted to a finite number of terms, while, for consistency with the classical theory, the constants must satisfy the requirement

$$
\sum_{i=1}^{M} \mu_{i} \alpha_{i}=2 \mu
$$

where $M$ is a positive integer and $\mu$ is again the shear modulus of the material in the undeformed stress-free (natural) configuration, as identified in (8).

We shall also make use of the strain energy expressed as a function of invariants (symmetric functions of the stretches). In particular, we mention here the representation of $W$ in terms of the two independent invariants $I_{1}$ and $I_{2}$ defined in terms of the stretches by

$I_{1}=\lambda_{1}^{2}+\lambda_{2}^{2}+\lambda_{3}^{2}, \quad I_{2}=\lambda_{1}^{-2}+\lambda_{2}^{-2}+\lambda_{3}^{-2}$.

Let $W$, regarded as a function of $I_{1}, I_{2}$, be written $\bar{W}\left(I_{1}, I_{2}\right)$. Then, we have the identification

$$
W\left(\lambda_{1}, \lambda_{2}, \lambda_{3}\right) \equiv \bar{W}\left(I_{1}, I_{2}\right) \text {. }
$$

In terms of $\bar{W}$ the principal Cauchy stress differences (7) become

$$
\begin{aligned}
& \sigma_{1}-\sigma_{3}=2\left(\lambda_{1}^{2}-\lambda_{1}^{-2} \lambda_{2}^{-2}\right) \frac{\partial \bar{W}}{\partial I_{1}}+2\left(\lambda_{1}^{2} \lambda_{2}^{2}-\lambda_{1}^{-2}\right) \frac{\partial \bar{W}}{\partial I_{2}}, \\
& \sigma_{2}-\sigma_{3}=2\left(\lambda_{2}^{2}-\lambda_{1}^{-2} \lambda_{2}^{-2}\right) \frac{\partial \bar{W}}{\partial I_{1}}+2\left(\lambda_{1}^{2} \lambda_{2}^{2}-\lambda_{2}^{-2}\right) \frac{\partial \bar{W}}{\partial I_{2}} .
\end{aligned}
$$

Analogues of the conditions (8) may be written down in respect of $\bar{W}$ but we omit the details.

Many specific forms of strain energy may be found in the literature, expressed either in terms of the stretches or the invariants $I_{1}$ and $I_{2}$ or other choices of independent deformation variables. For representative reviews of many of these we refer to, for example, Ogden [3, 7, 9], Treloar [10], Beatty [11] and Boyce and Arruda [12].

\section{3}

\section{Outline of the optimization method}

The experimental data used in this paper are the classical data of Jones and Treloar [2] for the biaxial tension data and of Treloar [1] for the other tests, their numerical values having been obtained from the original experimental tables. These data have been used to identify the parameters in specific strain-energy functions by means of the least squares (LS) technique. It should, however, be pointed out that sometimes in the literature these data are obtained by digitization of the figures in [1] and [2], with consequent loss of accuracy.

Let $\boldsymbol{\Lambda}=\left[\Lambda_{1}, \Lambda_{2}, \ldots, \Lambda_{m}\right]^{\mathrm{T}}$ be the vector of values of a measure of stretch appropriate to the considered deformation and $\tau=\left[\tau_{1}, \tau_{2}, \ldots, \tau_{m}\right]^{\mathrm{T}}$ be the corresponding values of stress, which may be either nominal stress or Cauchy stress, again depending on the deformation in question. Hence $(\boldsymbol{\Lambda}, \tau)$ are the given pairs of data values. The considered material model is represented by the strain-energy function $W$, from which the stress is calculated and written $F(\Lambda, \mathbf{p}): \mathbb{R} \times \mathbb{R}^{\mathbb{N}} \rightarrow \mathbb{R}$, where $\Lambda$ takes values $\Lambda_{i}, 1=1, \ldots, m$, and $\mathbf{p}=\left[p_{1}, p_{2}, \ldots, p_{n}\right]^{\mathrm{T}}$ is a set of $n$ material parameters (constants) to be identified.

Let us define the objective function as the squared 2-norm

$S_{F}(\mathbf{p}):=\|\mathbf{F}(\boldsymbol{\Lambda}, \mathbf{p})-\tau\|_{2}^{2}=\sum_{i=1}^{m}\left[F\left(\Lambda_{i}, \mathbf{p}\right)-\tau_{i}\right]^{2}$,

where $\mathbf{F}(\boldsymbol{\Lambda}, \mathbf{p}):=\left[F\left(\Lambda_{1}, \mathbf{p}\right), \ldots, F\left(\Lambda_{m}, \mathbf{p}\right)\right]^{\mathrm{T}}$.

Hence, the minimization problem is given by $\min _{\mathbf{p}} S_{F}(\mathbf{p})$.

If $F(\Lambda, \mathbf{p})$ is a non-linear function with respect to $\mathbf{p}$, then a non-linear least squares (NLS) problem arises.

Several numerical algorithms have been used in the literature to solve NLS problems (see, for example, Bjorck [13]) and they are usually based on some suitable modification of the Newton method and require an initial guess for the solution. Therefore, when some appropriate stopping criteria are satisfied, the iterative technique approximates numerically a minimum for the problem (20) and then furnishes an optimal solution, $\mathbf{p}^{*}$ say, for the fitting problem with residual $S \equiv S_{F}\left(\mathbf{p}^{*}\right)$.

For our purposes in the present paper we use the function Lsqcurvefit in the Optimization Toolbox of MATLAB [14]. We choose the trust region (TR) method (see [14] for details) as the option for the descent algorithm and, in general, we choose a randomly generated starting point. The iterations of the NLS algorithm can stop either when the Newton step becomes less than TolX $=1 \mathrm{e}-8$ or the infinity norm of the estimated gradient $\nabla$ of the objective function is less than TolFun $=1 \mathrm{e}-12$ or when a maximum of 3000 iterations have been performed. We remark that the value of $\nabla$ for each approximation obtained can be regarded as an indicator of the attainment of a local minimum for the NLS function. 
We did not impose upper and lower bounds on the parameters $\mathbf{p}$ since we chose to use an unconstrained optimization algorithm and to discard non-physical solutions a posteriori.

Finally, to allow comparisons between the different forms of $F(\Lambda, \mathbf{p})$, we report also the relative errors in the data obtained for the corresponding optimal numerical fitting and calculated as

$\operatorname{err}_{i}=\frac{\left|F\left(\Lambda_{i}, \mathbf{p}^{*}\right)-\tau_{i}\right|}{\max \left\{0.5,\left|\tau_{i}\right|\right\}} \quad$ for $i=1, \ldots, m$.

This is a slight modification of the usual definition in that the 0.5 has been included in the denominator so as to avoid division by small values of $\left|\tau_{i}\right|$ when the stretches are close to 1 .

Moreover, in order to estimate the predictive ability of each model, we propose a suitable modification of the above MATLAB function so as to fit several different sets of data together. For example, if the simple tension $\left(\boldsymbol{\Lambda}_{s}, \tau_{s}\right)$ and the equibiaxial tension data $\left(\boldsymbol{\Lambda}_{\mathrm{e}}, \tau_{\mathrm{e}}\right)$ are considered together we choose to minimize the new objective function

$$
\min _{\mathbf{p}}\left(\left\|\mathbf{F}_{\mathrm{s}}\left(\boldsymbol{\Lambda}_{\mathrm{s}}, \mathbf{p}\right)-\tau_{\mathrm{s}}\right\|_{2}^{2}+\left\|\mathbf{F}_{\mathrm{e}}\left(\boldsymbol{\Lambda}_{\mathrm{e}}, \mathbf{p}\right)-\tau_{\mathrm{e}}\right\|_{2}^{2}\right),
$$

where $\mathbf{F}_{\mathrm{s}}\left(\boldsymbol{\Lambda}_{\mathrm{s}}, \mathbf{p}\right)=\left[F_{\mathrm{s}}\left(\Lambda_{\mathrm{s} 1}, \mathbf{p}\right), \ldots, F_{\mathrm{s}}\left(\Lambda_{\mathrm{s} m}, \mathbf{p}\right)\right]^{\mathrm{T}}$, with $\Lambda_{s i}, i=1, \ldots, m$, being the entries in the vector $\Lambda_{\mathrm{s}}$ and $F_{\mathrm{s}}$ the appropriate form of $F$ for simple tension. Similarly for $F_{e}\left(\Lambda_{e}, p\right)$, except that the number of data pairs (i.e. the value of $m$ ) may be different. More details will be given in each section dedicated to specific models under examination.

We mention here that the units used in Treloar [1] are kg $\mathrm{cm}^{-2}$ and those in Jones and Treloar [2] are $\mathrm{N} \mathrm{mm}^{-2}$. We use these original units without further reference throughout the following sections, and we emphasize that the tests in these two papers were carried out for different materials.

\section{4}

\section{The Ogden model}

In this section we focus on the Ogden model identified in (14). Considerations of physically realistic response and material stability lead to the inequalities

$\mu_{i} \alpha_{i}>0$,

for each $i=1, \ldots, M$ (no sum over $i$ ) in addition to the connection (15). Note, however, that, in general, if $M \geq 3$ it is not necessary that these hold for every $i$. This model is most commonly implemented with $M=3$ or $M=4$, and the theoretical predictions of such a model are generally very satisfactory, at least from a qualitative point of view. Based on the same set of data as used here, the paper by Twizell and Ogden [15] is dedicated to fitting (14) by means of the Levenberg-Marquardt algorithm.

In the following subsections we shall examine this model in order to show that, given a set of experimental data, the following conclusions can be drawn:

- it is possible to find a large number of optimal sets of parameters;

- the number of the optimal sets of parameters and the values of the parameters depend on $M$ and also in a very sensitive way on the numerical accuracy required in the
NLS algorithm used (maximum number of iterations permitted, tolerance errors, etc.);

- the effect of this multiplicity of optimal parameters on the solution of a boundary-value problem is not negligible from the quantitative point of view.

\section{1}

\section{Simple tension}

In [15] the set of optimal parameters obtained in order to fit Treloar's simple tension experiment data [1] are

$\begin{array}{ccc}i & \alpha_{i} & \mu_{i} \\ 1 & 2.26 & 2.22 \\ 2 & -2.01 & -0.448 \\ 3 & 10.01 & 3.9 \mathrm{e}-7\end{array}$

for $M=3$ and

$\begin{array}{ccc}i & \alpha_{i} & \mu_{i} \\ 1 & 1.23 & 6.27 \\ 2 & -2.99 & -0.054 \\ 3 & 4.44 & 0.036 \\ 4 & 19.49 & 0.8 \mathrm{e}-15\end{array}$

for $M=4$. The analysis is based on equations equivalent to (11) with

$W_{s}=\sum_{i=1}^{M} \frac{\mu_{i}}{\alpha_{i}}\left(\lambda^{\alpha_{i}}+2 \lambda^{-\alpha_{i} / 2}-3\right)$,

which is the appropriate specialization of (14) for simple tension. For the set of parameters $(23)(M=3)$ the residual $S_{(23)}$ is found to be 13.449 , and from Fig. 1, in which the relative errors are plotted as functions of the stretch $\lambda$, it is clear that the relative error may be very large for low values of the stretch and moderate around $\lambda=4$. For the set of parameters $(24)(M=4)$ the residual $S_{(24)}$ is lowered to 8.0129 and Fig. 1 shows that the relative error is

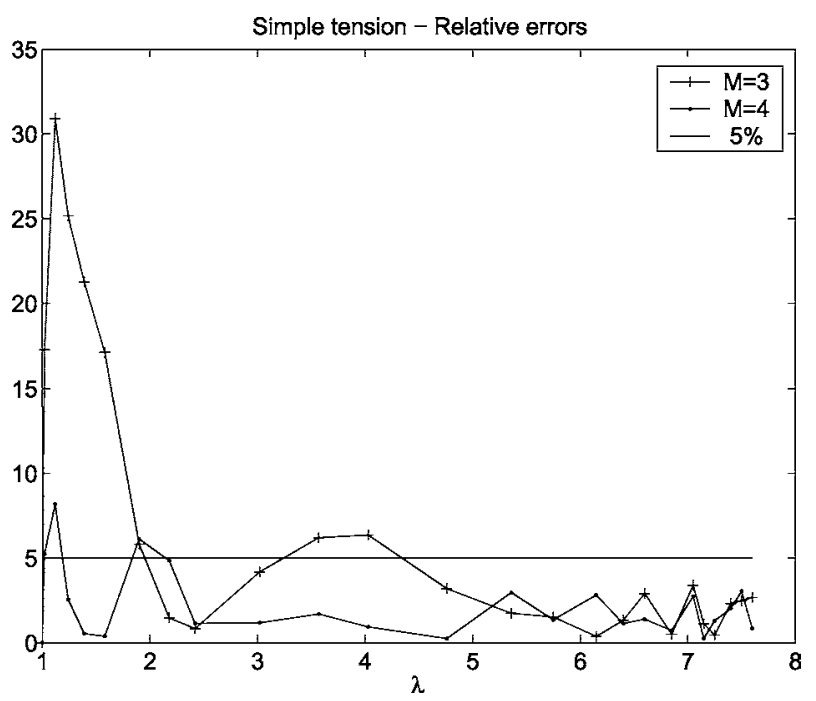

Fig. 1. Plots of the relative errors (\%) against stretch $\lambda$ in the simple tension data fits of the Twizell-Ogden parameter sets for $M=3,4$ 
Table 1. Parameter values for the Ogden model with $M=3$ : simple tension

\begin{tabular}{|c|c|c|c|c|c|c|c|c|}
\hline \multirow[t]{2}{*}{$i$} & \multicolumn{2}{|l|}{ OT } & \multicolumn{2}{|l|}{$\mathrm{OSS}_{1}$} & \multicolumn{2}{|l|}{$\mathrm{OSS}_{2}$} & \multicolumn{2}{|l|}{$\mathrm{OSS}_{3}$} \\
\hline & $\alpha_{i}$ & $\mu_{i}$ & $\alpha_{i}$ & $\mu_{i}$ & $\alpha_{i}$ & $\mu_{i}$ & $\alpha_{i}$ & $\mu_{i}$ \\
\hline 2 & -2.054 & -0.61139 & 3.2481 & 0.25031 & 2.845 & 0.63604 & -4.5926 & -1.2037 \\
\hline 3 & 10.01 & $3.9204 \mathrm{e}-7$ & -22.522 & $-2.5625 e-8$ & -2.7138 & -2.7391 & -20.594 & $-2.1212 \mathrm{e}-7$ \\
\hline$S$ & 12.876 & & 9.3318 & & 9.9655 & & 11.1 & \\
\hline
\end{tabular}

very low across the range of values of the stretch except for limited ranges where it is moderate.

Let us focus on the case $M=3$ and use the set (23) together with 30 sets of random values as starting guesses for the NLS algorithm. The algorithm converges to the set of parameters reported in Table 1, where for each optimal set $\mathbf{p}^{*}=\left[\alpha_{1}, \mu_{1}, \ldots, \alpha_{M}, \mu_{M}\right]^{\mathrm{T}}$ we report also the residual $S \equiv S\left(\mathbf{p}^{*}\right)$ and the measure of the gradient $\nabla$, as explained in Sect. 3.

By OT we identify the solution obtained from our algorithm by starting from (23) with at most four significant digits and by $\operatorname{OSS}_{i}, i=1,2,3$, the other possible solutions obtained by starting from the random guesses. By using a larger number of random guesses it is possible to find other solutions. In this case, starting from the (23) guess, we find a set of parameters very similar to those in (23), but the new solution OT improves the fit, as illustrated by the distribution of the relative errors shown in Fig. 2, and, in particular, it should be noted that $\mathrm{OSS}_{2}$ gives the lowest relative error.

We repeat this procedure for the case $M=4$. The results are reported in Table 2 and the relative errors in Fig. 3. Note that the $\mathrm{OSS}_{2}$ set of parameters gives theoretical predictions with all the relative errors under the $5 \%$ level.

As a general comment on the Ogden model, Tables 1 and 2 give evidence of the multiple choices encountered.

To emphasize that application of the NLS method to the Ogden function (14) can be very sensitive to the numerical settings used in the implementation of the algorithm, we report the following considerations. In the numerical experiments above, we found that the NLS optimization often stops when the Newton step becomes less than TolX $=1 \mathrm{e}-8$ even if neither the estimated gradient $\nabla$ nor the residual $S$ is sufficiently small. Moreover, if we set TolX $=1 \mathrm{e}-15$ up to the machine precision and we restart the algorithm from one solution $\mathbf{p}^{*}$ obtained when TolX $=1 \mathrm{e}-8$ is used, sometimes a completely different set of parameters with a lower residual can be obtained. For example, if we choose $\mathbf{p}^{*} \equiv \mathrm{OT}$ in Table 1 as a new starting guess, then the new set

$$
\begin{array}{ccc}
i & \alpha_{i} & \mu_{i} \\
1 & 2.7908 & 0.81107 \\
2 & -1.6207 & -3.9647 \\
3 & 10.95 & 5.0211 \mathrm{e}-8
\end{array}
$$

with a residual $S=9.57949$ and $\nabla=0.504$ is obtained.

This behavior of the Newton iterations usually indicates that the problem becomes ill-conditioned, that is 'low' and 'flat' local minima can be present in the objective function of the optimization problem.

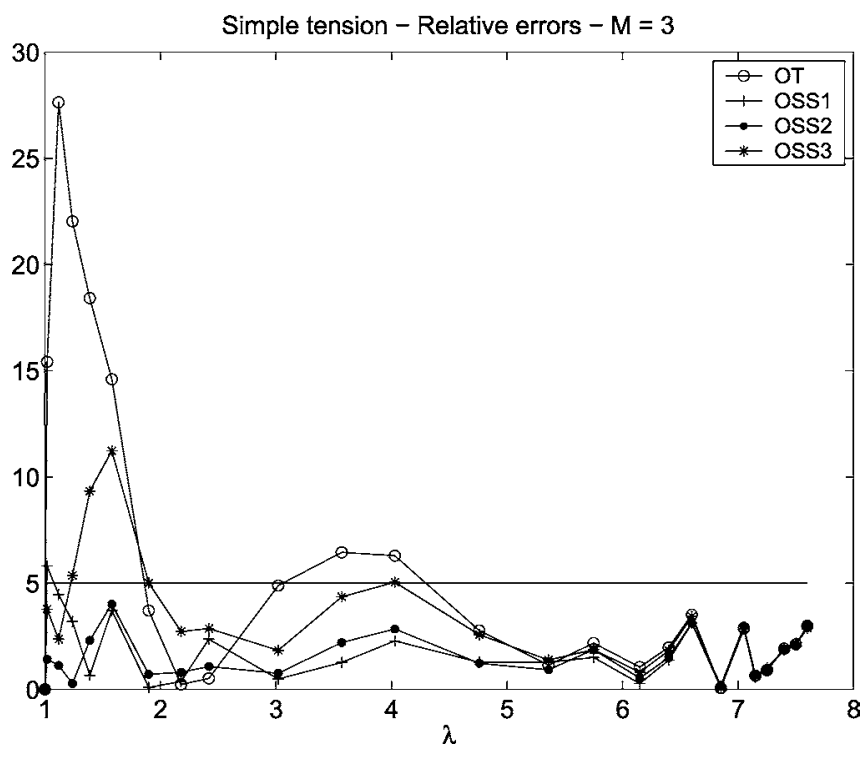

Fig. 2. Plots of the relative errors (\%) against stretch $\lambda$ in respect

\begin{tabular}{|c|c|c|c|c|c|c|c|c|}
\hline \multirow[t]{2}{*}{$i$} & \multicolumn{2}{|l|}{ OT } & \multicolumn{2}{|l|}{$\mathrm{OSS}_{1}$} & \multicolumn{2}{|l|}{$\mathrm{OSS}_{2}$} & \multicolumn{2}{|l|}{$\mathrm{OSS}_{3}$} \\
\hline & $\alpha_{i}$ & $\mu_{i}$ & $\alpha_{i}$ & $\mu_{i}$ & $\alpha_{i}$ & $\mu_{i}$ & $\alpha_{i}$ & $\mu_{i}$ \\
\hline 2 & -1.99 & -0.054 & -1.605 & -1.4396 & -2.0354 & -3.8145 & -12.161 & $-9.8765 e-4$ \\
\hline 3 & 4.44 & 0.036 & -1.6087 & -2.0189 & 13.945 & $9.5425 \mathrm{e}-11$ & -0.039294 & -43.239 \\
\hline 4 & 19.49 & $7.6559 e-16$ & 2.654 & 1.0229 & 3.2503 & 0.33457 & -4.3008 & -2.0949 \\
\hline
\end{tabular}
of the Ogden strain energy with $M=3$ in simple tension for the parameter sets OT, $\mathrm{OSS}_{1}, \mathrm{OSS}_{2}, \mathrm{OSS}_{3}$ given in Table 1

Table 2. Parameter values for the Ogden model with $M=4$ : simple tension 


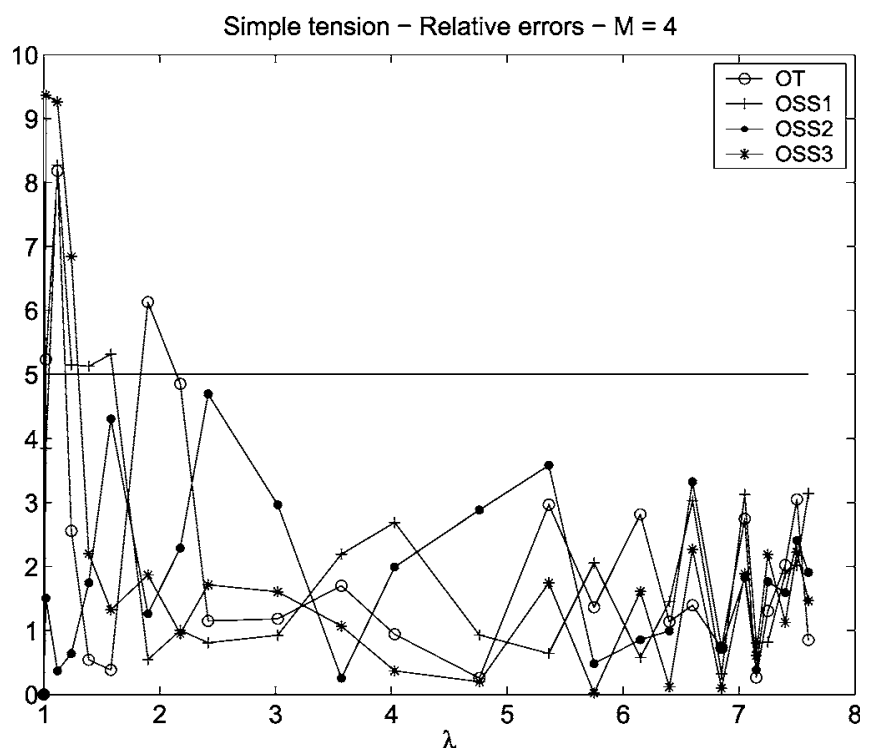

Fig. 3. Plots of the relative errors (\%) against stretch $\lambda$ in respect of the Ogden strain energy with $M=4$ in simple tension for the parameter sets OT, $\mathrm{OSS}_{1}, \mathrm{OSS}_{2}, \mathrm{OSS}_{3}$ given in Table 2

\section{2}

\section{Sensitivity of the Ogden strain energy}

In the fittings discussed above we have noted that the relative errors are always higher in the region of small stretches. It is therefore interesting to perform a sensitivity analysis for the Ogden strain-energy function (14) in the case of simple tension, in which case, with $\lambda_{1}=\lambda, \lambda_{2}=\lambda_{3}=\lambda^{-1 / 2}$, we may write the energy as a function of $\lambda$, as in (25). The corresponding nominal stress $t_{\mathrm{s}}$ is then

$t_{\mathrm{s}}=\sum_{i=1}^{M} \mu_{i}\left(\lambda^{\alpha_{i}-1}-\lambda^{-\alpha_{i} / 2-1}\right) \equiv F_{\mathrm{s}}(\lambda, \mathbf{p})$.

For each $i$, the absolute sensitivities of the parameters $\alpha_{i}, \mu_{i}$ can be assessed by considering

$$
\begin{aligned}
\left|\frac{\partial t_{s}}{\partial \mu_{i}}\right| & =\left|\lambda^{\alpha_{i}-1}-\lambda^{-\alpha_{i} / 2-1}\right|, \\
\left|\frac{\partial t_{s}}{\partial \alpha_{i}}\right| & =\left|\mu_{i} \ln (\lambda)\left(\lambda^{\alpha_{i}-1}+\frac{1}{2} \lambda^{-\alpha_{i} / 2-1}\right)\right| .
\end{aligned}
$$

However, such quantities can be inappropriate because they are not invariant with respect to changes in the magnitude of either $t_{\mathrm{s}}$ or of one parameter. Relative changes in $t_{\mathrm{s}}$ with respect to a relative change in $\alpha_{i}$ or $\mu_{i}$ are provided, respectively, by

$$
\left|\frac{\mu_{i}}{t_{0}} \frac{\partial t_{\mathrm{s}}}{\partial \mu_{i}}\right|, \quad\left|\frac{\alpha_{i}}{t_{0}} \frac{\partial t_{\mathrm{s}}}{\partial \alpha_{i}}\right|,
$$

where $t_{0}$ is the value of $t_{\mathrm{s}}$ at some specified set of parameter values. For example, by considering $t_{\mathrm{s}}$ to be evaluated at the set (23) for $M=3$, we obtain, for $i=1, \ldots, 3$,

$$
\left|\frac{\mu_{i}}{t_{0}} \frac{\partial t_{s}}{\partial \mu_{i}}\right|=\frac{\left|\mu_{i}\left(\lambda^{\alpha_{i}-1}-\lambda^{-\alpha_{i} / 2-1}\right)\right|}{\left|t_{0}\right|}
$$

and

$$
\left|\frac{\alpha_{i}}{t_{0}} \frac{\partial t_{s}}{\partial \alpha_{i}}\right|=\frac{\left|\mu_{i} \ln (\lambda)\left(\lambda^{\alpha_{i}-1}+\frac{1}{2} \lambda^{-\alpha_{i} / 2-1}\right)_{\alpha_{i}}\right|}{\left|t_{0}\right|}
$$

where

$$
\begin{aligned}
t_{0}= & 2.22\left(\lambda^{1.26}-\lambda^{-2.13}\right)-0.448\left(\lambda^{-3.01}-\lambda^{0.005}\right) \\
& +3.9 \mathrm{e}-7\left(\lambda^{9.01}-\lambda^{-6.005}\right) .
\end{aligned}
$$

Since these functions are both linear in $\mu_{i}$, we fix $\mu_{i}=1$ and we plot them in Fig. 4 as functions of $\alpha_{i}$ and $\lambda$. We observe that all parameters are sensitive for stretches in the region $0.5 \leq \lambda \leq 1.5$ in each case. This could also explain why bigger relative errors are generally concentrated in this region. A similar analysis can be carried out for the other deformations considered in the following subsections.

\section{3}

\section{Equibiaxial deformations}

We now turn attention to equibiaxial deformations. The parameters reported in [15] that provide fits to the Treloar data are

$$
\begin{array}{ccc}
i & \alpha_{i} & \mu_{i} \\
1 & 1.46 & 5.39 \\
2 & -2.03 & -0.531 \\
3 & 9.68 & 0.19 \mathrm{e}-5
\end{array}
$$

for $M=3$, and

$\begin{array}{ccc}i & \alpha_{i} & \mu_{i} \\ 1 & 1.26 & 6.17 \\ 2 & -2.01 & -0.0091 \\ 3 & 4.26 & 0.046 \\ 4 & 19.49 & 1 \mathrm{e}-14\end{array}$

for $M=4$, and the relevant relationship between $\lambda$ and $t$ is given by (13) with (12), specialized in respect of (14).
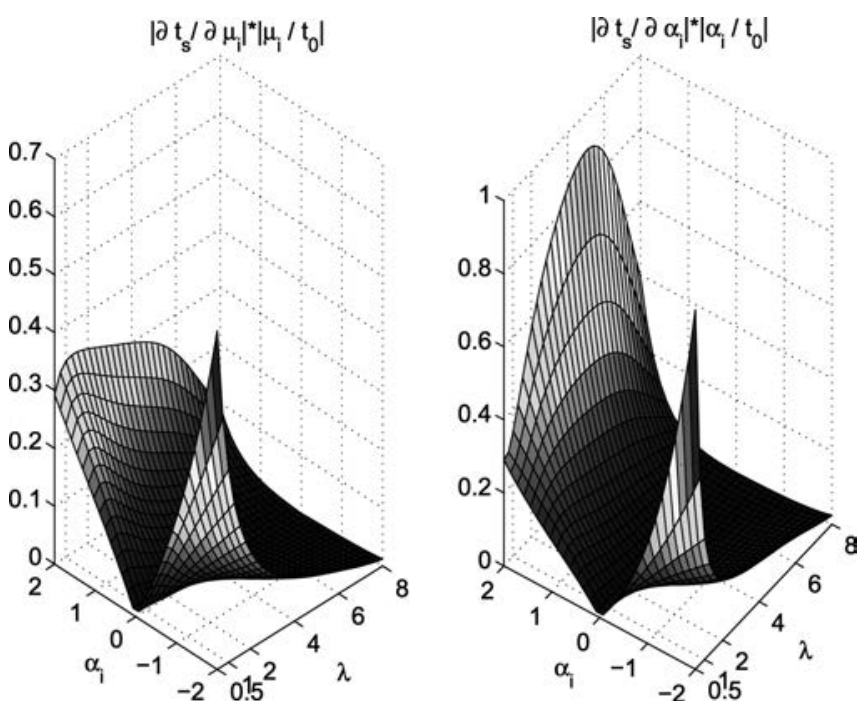

Fig. 4. Relative sensitivity of the Ogden parameters: plot of (28) (left) and (29) (right) against $\lambda$ and $\alpha_{i}$ 
Table 3. Parameter values for the Ogden model with $M=3$ : equibiaxial tension

\begin{tabular}{|c|c|c|c|c|c|c|}
\hline \multirow[t]{2}{*}{$i$} & \multicolumn{2}{|l|}{$\mathrm{OSS}_{1}$} & \multicolumn{2}{|l|}{$\mathrm{OSS}_{2}$} & \multicolumn{2}{|l|}{$\mathrm{OSS}_{3}$} \\
\hline & $\alpha_{i}$ & $\mu_{i}$ & $\alpha_{i}$ & $\mu_{i}$ & $\alpha_{i}$ & $\mu_{i}$ \\
\hline 1 & -6.7054 & $-4.0441 e-9$ & 1.6918 & 4.4184 & 4.259 & 0.13873 \\
\hline 2 & 1.58 & 5.1733 & 5.4929 & 0.013381 & -0.022671 & -144.69 \\
\hline 3 & -2.4591 & -0.03445 & 1.6919 & 0.47456 & -0.022671 & -142.01 \\
\hline$S$ & 0.31666 & & 0.30387 & & 0.70829 & \\
\hline$\nabla$ & 0.020419 & & $2.821 \mathrm{e}-5$ & & 0.0088361 & \\
\hline
\end{tabular}

Table 4. Parameter values for the Ogden model with $M=4$ : equibiaxial tension

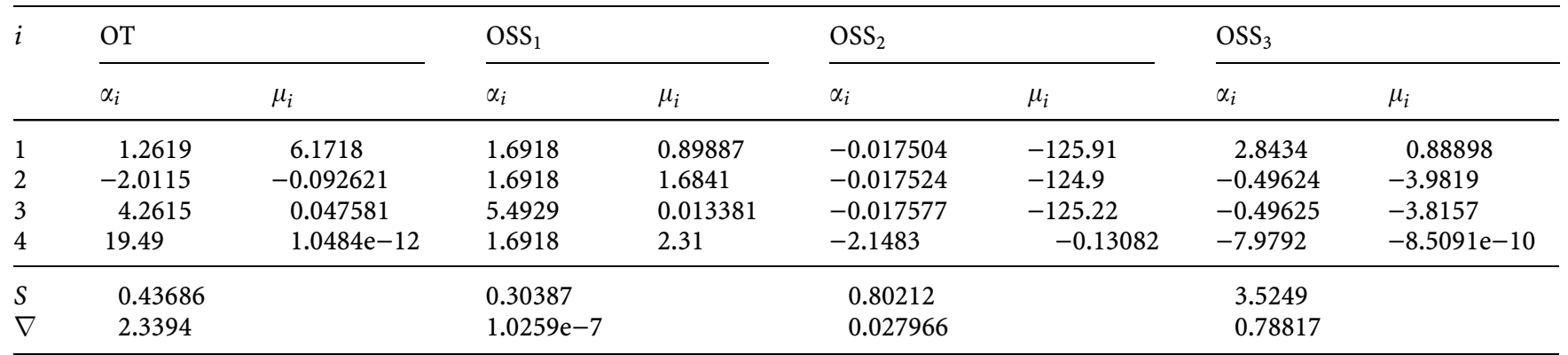

The set of parameters reported for $M=3$ is not considered since in this case the total residual is 4158.9 , while for $M=4$ it is 3.6391. The results obtained from our simulations are summarized in Tables 3 and 4, and the corresponding distributions of relative errors are shown in Figs. 5 and 6, respectively. Note that if the NLS technique starts with (30) then the solution obtained does not satisfy the condition (22) for every $i$.

\section{4}

\section{Simple and equibiaxial tension compared}

In this section we compare the fits for simple and equibiaxial tension by selecting those parameters in the previous tables that give the lowest relative error in the infinity norm for both sets of Treloar data.

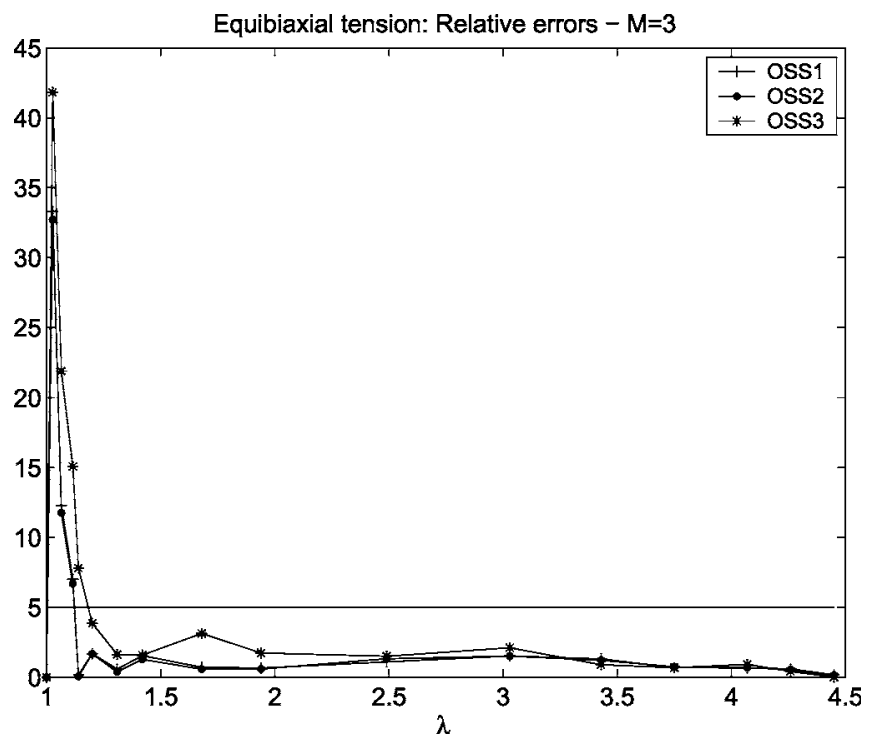

Fig. 5. Plots of the relative errors (\%) against stretch $\lambda$ in respect of the Ogden strain energy with $M=3$ in equibiaxial tension for the parameter sets $\mathrm{OSS}_{1}, \mathrm{OSS}_{2}, \mathrm{OSS}_{3}$ given in Table 3
Consider the case $M=3$. Among the optimal minima obtained for simple tension, the OT set in Table 1 produces the best fit (in the sense just specified) for the equibiaxial data. On the other hand, among the optimal minima obtained for equibiaxial tension, the $\mathrm{OSS}_{2}$ set in Table 3 produces the best fit for the simple tension data. These behaviours are reported in Figs. 7 and 8, respectively, and it easy to see that in each case no good agreement is obtained for both simple tension and equibiaxial tension simultaneously. The situation is only slightly improved if we take $M=4$, so we do not show the analogues of Figs. 7 and 8 for this case.

A better fit is obtained if we apply the NLS algorithm to both sets of Treloar data simultaneously, i.e. $\left(\boldsymbol{\Lambda}_{\mathrm{s}}, \tau_{\mathrm{s}}\right)$ for

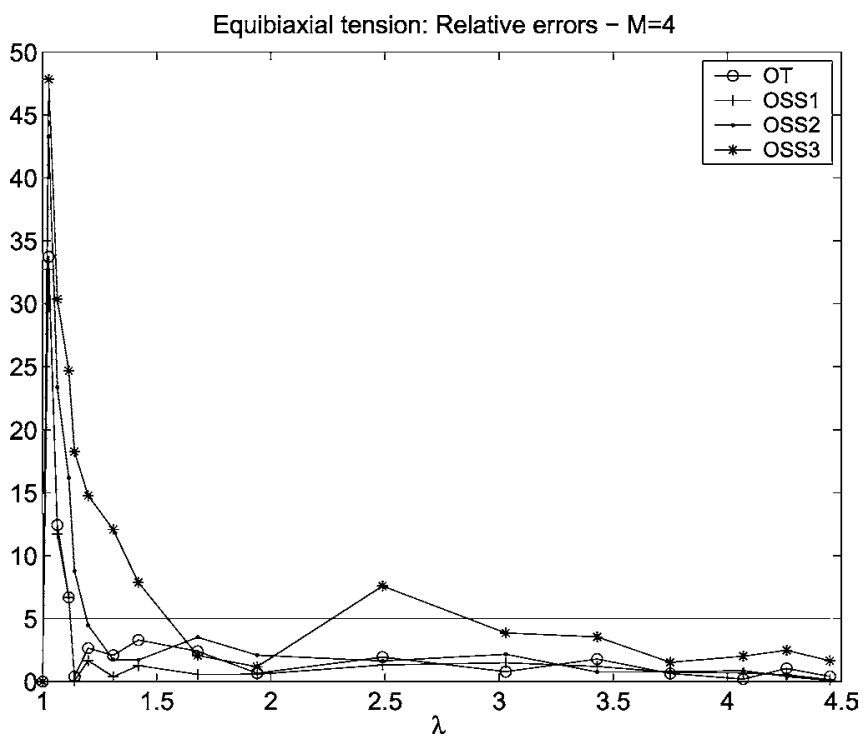

Fig. 6. Plots of the relative errors (\%) against stretch $\lambda$ in respect of the Ogden strain energy with $M=4$ in equibiaxial tension for the parameter sets OT, $\mathrm{OSS}_{1}, \mathrm{OSS}_{2}, \mathrm{OSS}_{3}$ given in Table 4 


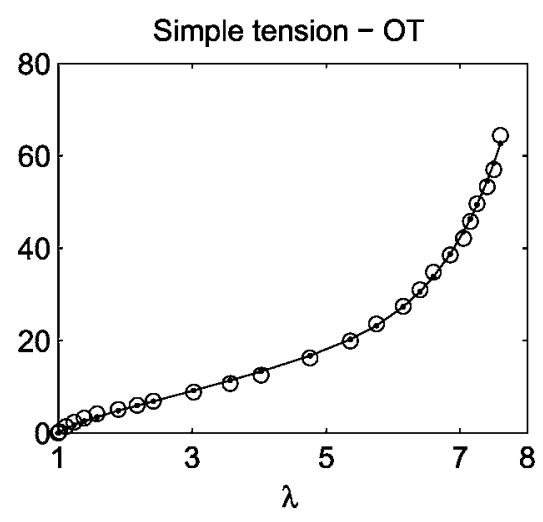

Equibiaxial tension
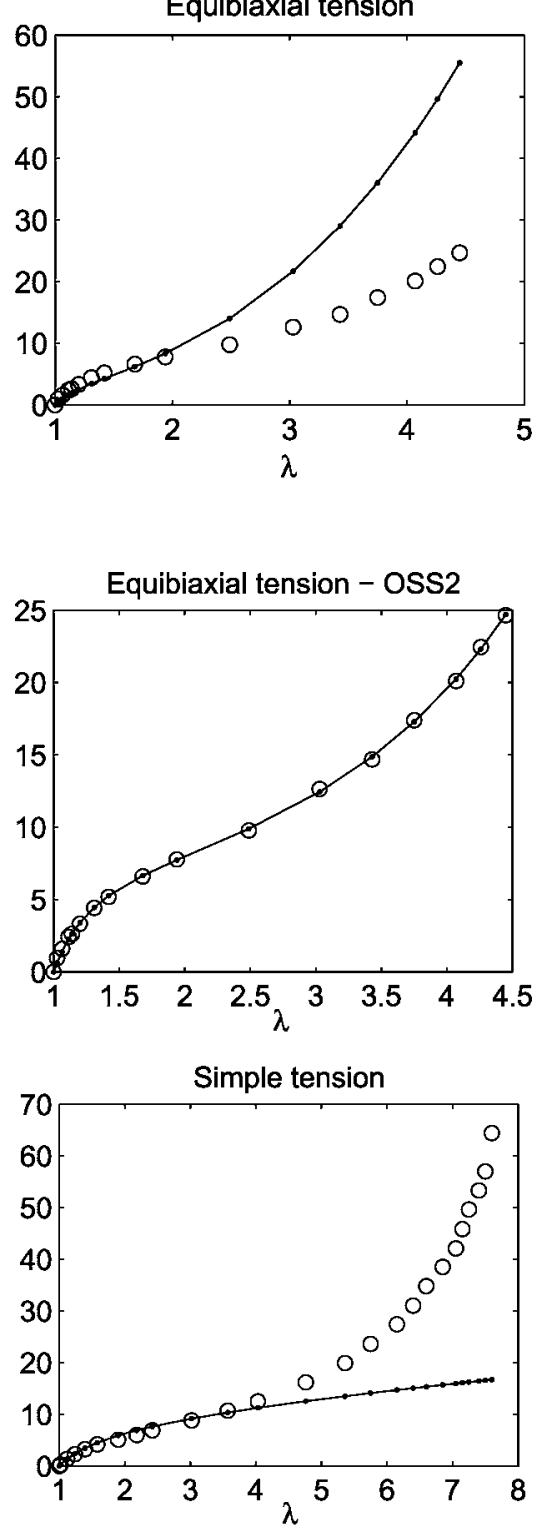
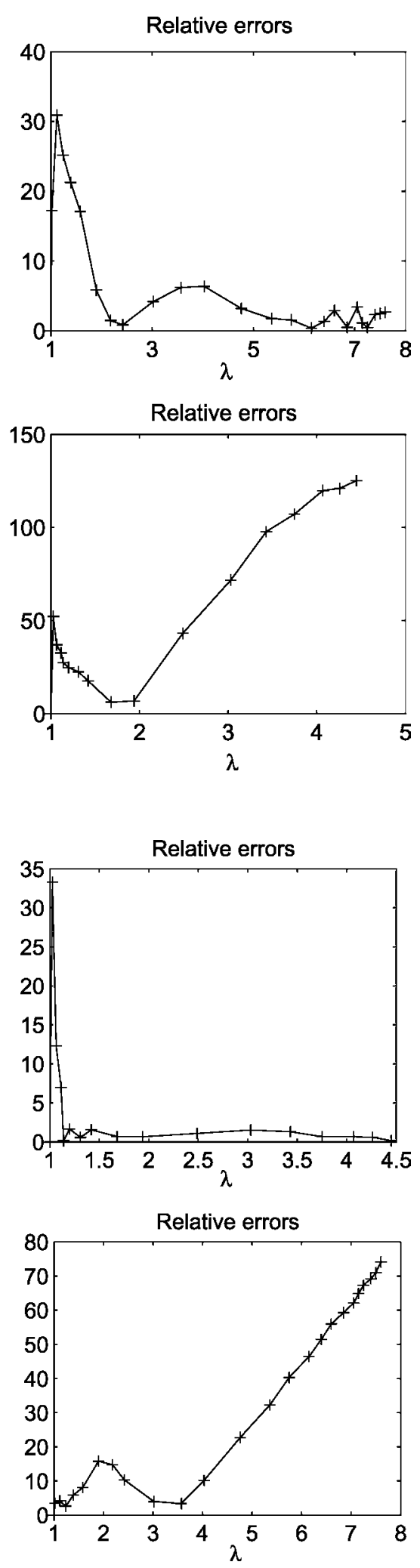

Fig. 7. Fit to the simple tension and equibiaxial tension data (circles) using the OT Table 1 parameter set obtained from the simple tension data, and the relative errors: $M=3$. In the left-hand (right-hand) figures nominal stresses (relative errors) are plotted against stretch
Fig. 8. Fit to the simple tension and equibiaxial tension data (circles) using the $\mathrm{OSS}_{2}-$ Table 3 parameter set obtained from the equibiaxial tension data, and the relative errors: $M=3$. In the left-hand (right-hand) figures nominal stresses (relative errors) are plotted against stretch simple tension and $\left(\boldsymbol{\Lambda}_{\mathrm{e}}, \tau_{\mathrm{e}}\right)$ for equibiaxial tension. In this case we optimize the objective function (21), where $\mathbf{p}=\left[\alpha_{1}, \mu_{1}, \ldots, \alpha_{M}, \mu_{M}\right]^{\mathrm{T}}$.

Consider $M=3$. By starting the algorithm with, for example, the optimal set OT - Table 1 and also from 10 random choices, the optimal set obtained is, in each case,

$$
\begin{array}{ccc}
i & \alpha_{i} & \mu_{i} \\
1 & 8.3952 & 1.2069 \mathrm{e}-5 \\
2 & 1.8821 & 3.7729 \\
3 & -2.2453 & -0.052171
\end{array}
$$

with $S=20.013, \nabla=0.0015682, S_{\mathrm{s}}=17.233, S_{\mathrm{e}}=2.7801$, where $S_{\mathrm{s}}$ and $S_{\mathrm{e}}$ are the residuals for the simple tension 

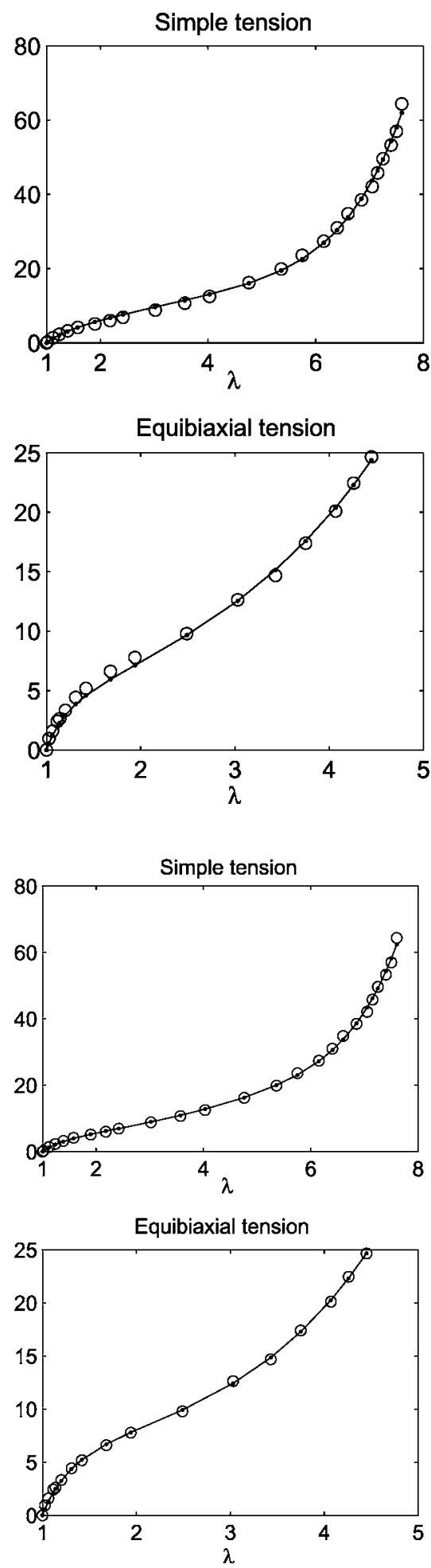
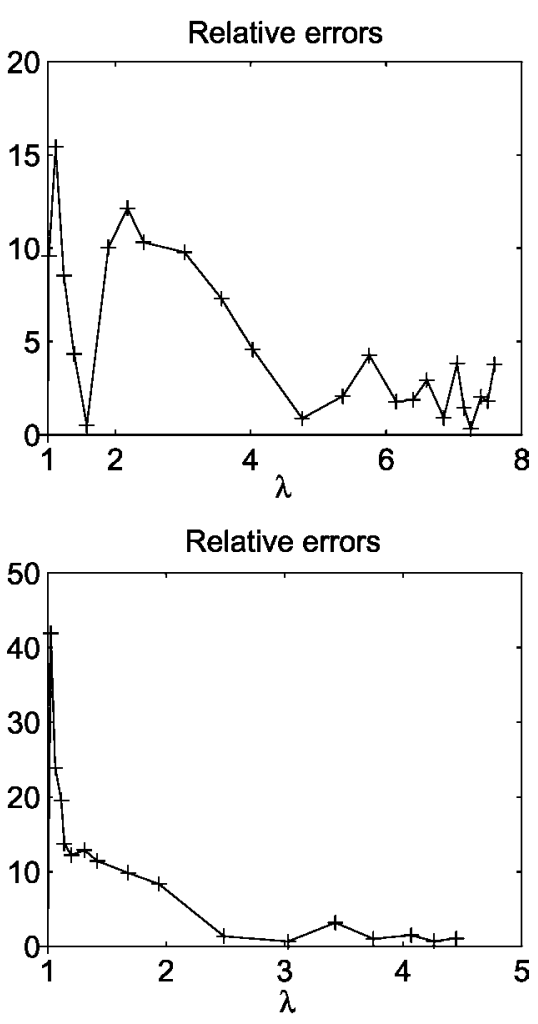

Fig. 9. Fits using both simple and equibiaxial tension data (circles) and the corresponding relative errors: $M=3$. In the left-hand (righthand) figures nominal stresses (relative errors) are plotted against stretch
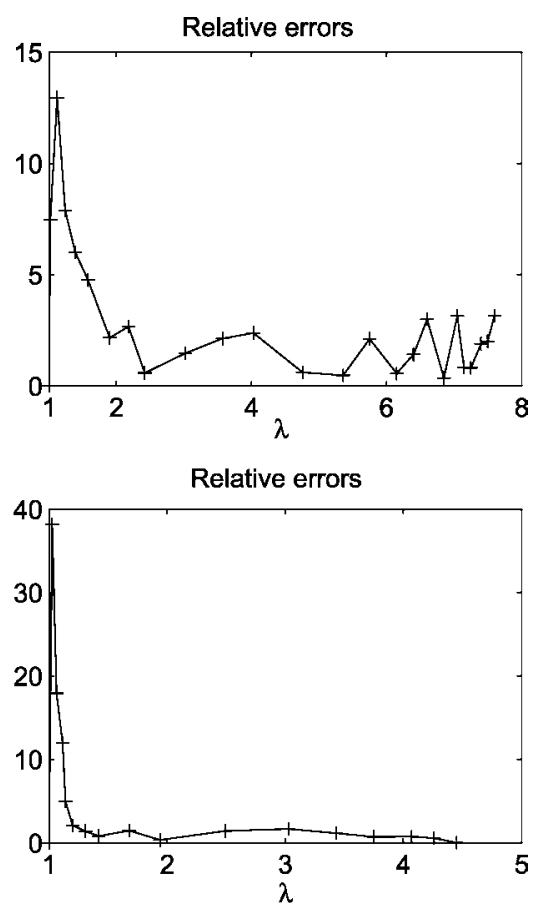

Fig. 10. Fits using both simple and equibiaxial tension data (circles) and the corresponding relative errors: $M=4$. In the lefthand (right-hand) figures nominal stresses (relative errors) are plotted against stretch and equibiaxial tension data separately. The corresponding fits and relative errors are shown in Fig. 9. Note that relative errors around the $10 \%$ level are obtained.

If $M=4$, the solution obtained is

$\begin{array}{ccc}i & \alpha_{i} & \mu_{i} \\ 1 & 2.7971 & 0.77817 \\ 2 & -2.7188 & -0.011229 \\ 3 & 10.505 & 1.269 \mathrm{e}-7 \\ 4 & 0.33382 & 16.169\end{array}$

and in this case we have $S=10.904, \nabla=1.3192$, $S_{\mathrm{s}}=10.427, S_{\mathrm{e}}=0.4765$, and the simple tension and equibiaxial tension fits are both shown in Fig. 10 along with the relative errors.

\section{5}

\section{Biaxial data}

As discussed in Ogden [6] as well as in the book [9], the set $\mathbf{p}_{\text {og }}$ given by 
Table 5. Parameter values for the Ogden model with $M=3$ : biaxial deformation

\begin{tabular}{|c|c|c|c|c|c|c|}
\hline \multirow[t]{2}{*}{$i$} & \multicolumn{2}{|l|}{ OG } & \multicolumn{2}{|l|}{$\mathrm{OSS}_{1}$} & \multicolumn{2}{|l|}{$\mathrm{OSS}_{2}$} \\
\hline & $\alpha_{i}$ & $\mu_{i}$ & $\alpha_{i}$ & $\mu_{i}$ & $\alpha_{i}$ & $\mu_{i}$ \\
\hline 1 & 1.2921 & 0.7332 & 6.8269 & $3.8409 \mathrm{e}-4$ & 4.5686 & 0.0048952 \\
\hline 2 & 4.5537 & 0.0049951 & 1.3193 & 0.73974 & 1.2935 & 0.73275 \\
\hline 3 & -2.4381 & -0.0052363 & -2.6059 & -0.0040178 & -2.4363 & -0.0052583 \\
\hline$S_{\min }$ & 0.0064745 & & 0.004571 & & 0.0064744 & \\
\hline$S_{\max }$ & 0.061694 & & 0.051234 & & 0.06157 & \\
\hline$\nabla$ & $2.0927 \mathrm{e}-6$ & & $3.6893 e-6$ & & $5.63 e-7$ & \\
\hline
\end{tabular}

$\begin{array}{ccc}i & \alpha_{i} & \mu_{i} \\ 1 & 1.3 & 0.69 \\ 2 & 4 & 0.01 \\ 3 & -2 & -0.0122\end{array}$

provides a good prediction of the biaxial data for the material tested by Jones and Treloar [2], which is a different material from that used by Treloar [1] so that crosscorrelation between the two sets of data is not admissible. The basis for the fitting in this case is the stress difference $\sigma_{1}-\sigma_{2}$, calculated from (7) and specialized for (14), plotted against $\lambda_{1}$ for a series of fixed values of $\lambda_{2}$. The relevant function $F(\lambda, \mathbf{p})$ is given by

$F\left(\lambda_{1}, \mathbf{p}\right)=\sum_{i=1}^{M} \mu_{i}\left(\lambda_{1}^{\alpha_{i}}-\lambda_{2}^{\alpha_{i}}\right)$,

with $\mathbf{p}=\left[\mu_{1}, \alpha_{1}, \ldots, \mu_{M}, \alpha_{M}\right]^{\mathrm{T}}, M=3$ and $\lambda_{2}$ fixed. We estimate that $\mathbf{p}_{\text {og }}$ produces a minimum residual
$S_{\min }=0.026728$ and a maximum residual $S_{\max }=0.035938$ on the biaxial data amongst the different values of $\lambda_{2}$.

In our simulation, the NLS algorithm is applied for each set of data with $\lambda_{2}$ fixed and then predictions for the sets of data with other values of $\lambda_{2}$ are derived using the optimal set of parameters obtained. Also, for the biaxial data, we show that the Ogden strain-energy function gives good fits with several different optimal sets of parameters. In fact, by starting the NLS procedure from $\mathbf{p}_{\text {og }}$, we find the set reported as OG in Table 5, while starting with 30 random samples different local minima are obtained. Some of these sets of parameters are shown in Table 5 together with the corresponding minimum and maximum residuals between the different values of $\lambda_{2}$. In Fig. 11 we show the fits obtained using the $\mathrm{OSS}_{1}$ set (which has the lowest residuals) and the corresponding relative errors.

If the Ogden strain energy with $M=4$ is considered, then the optimal sets in Table 6 are obtained, but the

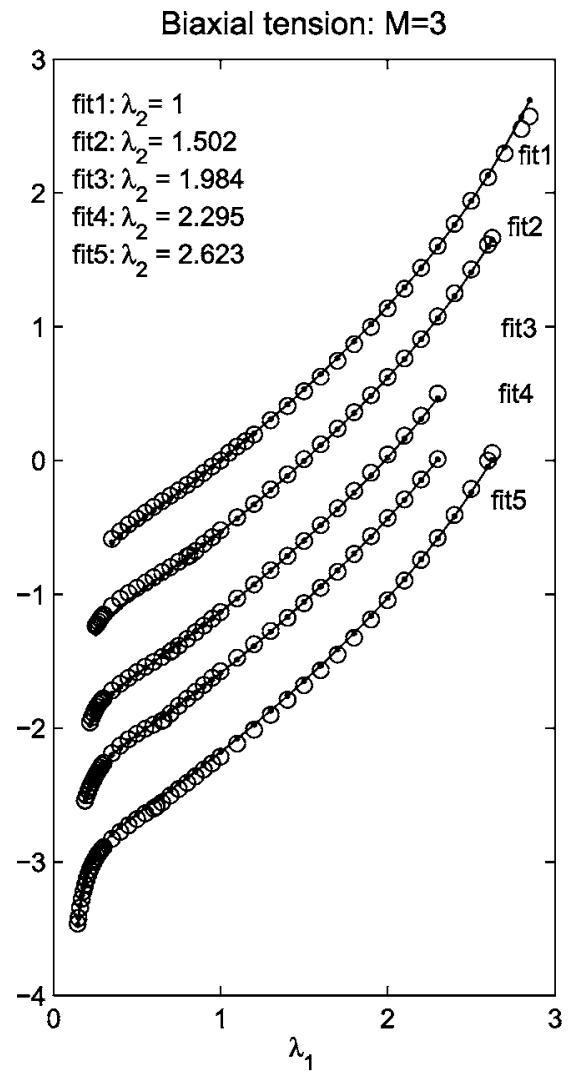

Fig. 11. Fit of the biaxial data (circles) using the Ogden strain energy for the optimal set $\mathrm{OSS}_{1}$ in Table 5, and the corresponding relative errors: $M=3$. In the left-hand figure $\sigma_{1}-\sigma_{2}$ is plotted against $\lambda_{1}$ for the fixed values of $\lambda_{2}$ indicated 
relative errors are not improved compared with the case of $M=3$.

Table 6. Parameter values for the Ogden model with $M=4$ : biaxial deformation

\begin{tabular}{|c|c|c|c|c|}
\hline \multirow[t]{2}{*}{$i$} & \multicolumn{2}{|l|}{$\mathrm{OSS}_{1}$} & \multicolumn{2}{|l|}{$\mathrm{OSS}_{2}$} \\
\hline & $\alpha_{i}$ & $\mu_{i}$ & $\alpha_{i}$ & $\mu_{i}$ \\
\hline 1 & -0.0067956 & -15.013 & -0.0079652 & -17.824 \\
\hline 2 & -3.3132 & $-8.0266 \mathrm{e}-4$ & -3.4693 & $4.5919 \mathrm{e}-4$ \\
\hline 3 & 1.7426 & 0.43689 & 2.0035 & 0.3301 \\
\hline 4 & -0.0067961 & -15.284 & -0.0079625 & -18.026 \\
\hline$S_{\min }$ & 0.0058526 & & 0.00948 & \\
\hline$S_{\max }$ & 0.53747 & & 0.12028 & \\
\hline$\nabla$ & $1.1627 \mathrm{e}-5$ & & $3.2675 e-4$ & \\
\hline
\end{tabular}

\section{5}

\section{Limiting chain extensibility models}

In order to contrast with the results in Sect. 4 we now consider the strain energy introduced by Pucci and Saccomandi [4], which we referred to in Sect. 1 as the Pucci-Saccomandi model. The Gent model itself reflects, at the phenomenological level, the behaviour of molecular models based on Langevin statistics. In particular, it represents very well the stiffening of the material at large strains. It has the advantage of mathematical simplicity and allows detailed analysis and explicit solution of particular boundary-value problems. Since the Pucci-Saccomandi model is a modification of the Gent model [16] with the addition of a logarithmic term similar to that in the Gent model we also refer to it as the 'Gent + Gent' model.
We use the abbreviation GG to identify the model, which has the form

$$
\begin{aligned}
\bar{W}\left(I_{1}, I_{2}\right)= & -\frac{\mu}{2} J_{m} \log \left(1-\frac{I_{1}-3}{J_{m}}\right) \\
& +C_{2} \log \left(\frac{I_{2}}{3}\right),
\end{aligned}
$$

where $J_{m}$ is a positive material constant that identifies the upper limit of $I_{1}$ (i.e. $I_{1}<3+J_{m}$ ) associated with limiting chain extensibility in the molecular-based models, $C_{2}$ is another material constant (associated with the contribution of $I_{2}$ ) and we recall the expressions (16) for the invariants $I_{1}$ and $I_{2}$, which are subject to (3). Note that $\mu+2 C_{2} / 3$ is the infinitesimal shear modulus and that the original Gent model corresponds to $C_{2}=0$. The associated principal stress differences may be calculated from the formulas (18), but we do not give them explicitly here.

Table 7. Parameter values from the fit of the GG model to simple tension and equibiaxial tension data

\begin{tabular}{llllll}
\hline & $\mu$ & $J_{m}$ & $C_{2}$ & $S$ & $\nabla$ \\
\hline Simple tension & 2.4195 & 77.931 & 1.0233 & 7.6082 & $4.4123 \mathrm{e}-5$ \\
Equib. tension & 3.1674 & 88.13 & 0.43659 & 0.36221 & $8.1682 \mathrm{e}-8$ \\
\hline
\end{tabular}

By fitting the GG model for the simple tension and equibiaxial tension data we obtain the sets of parameters in Table 7. In Figs. 12 and 13 we show that each set exhibits a valuable predictive property since relative errors below $20 \%$ are generated when the parameters obtained by the simple tension (equibiaxial tension) fit are used to compare the theory with the data for equibiaxial tension
GG model: Simple tension

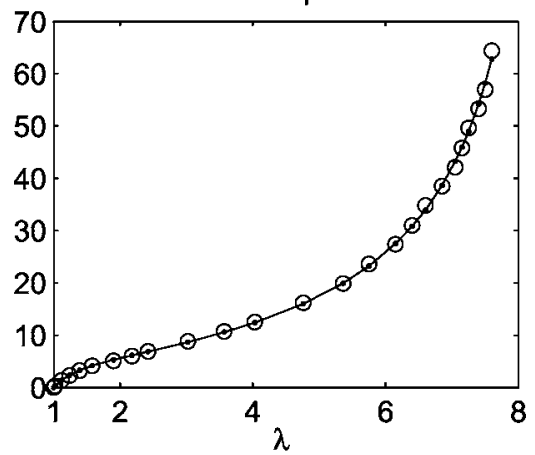

Equibiaxial tension

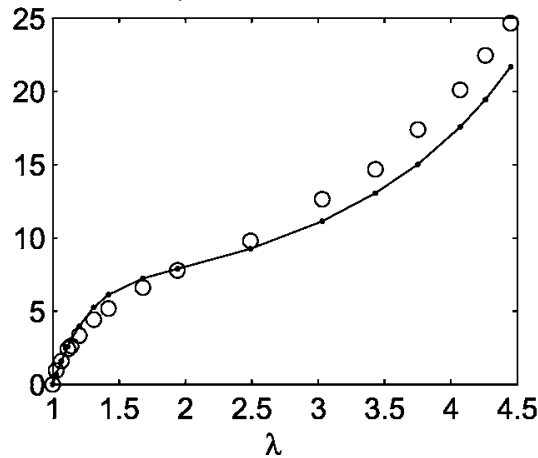

Relative errors

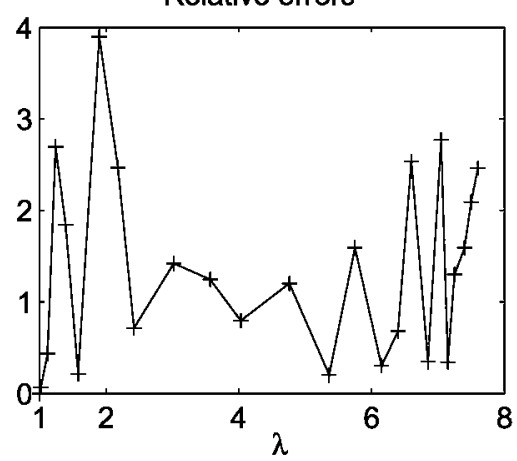

Relative errors

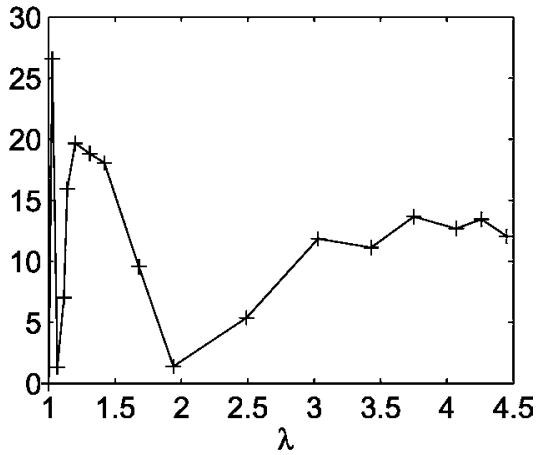

Fig. 12. Fit of the GG model to simple tension and equibiaxial tension data (circles) and the corresponding relative errors with the optimal parameter set based on use of the simple tension data. In the left-hand (right-hand) figures nominal stresses (relative errors) are plotted against stretch 

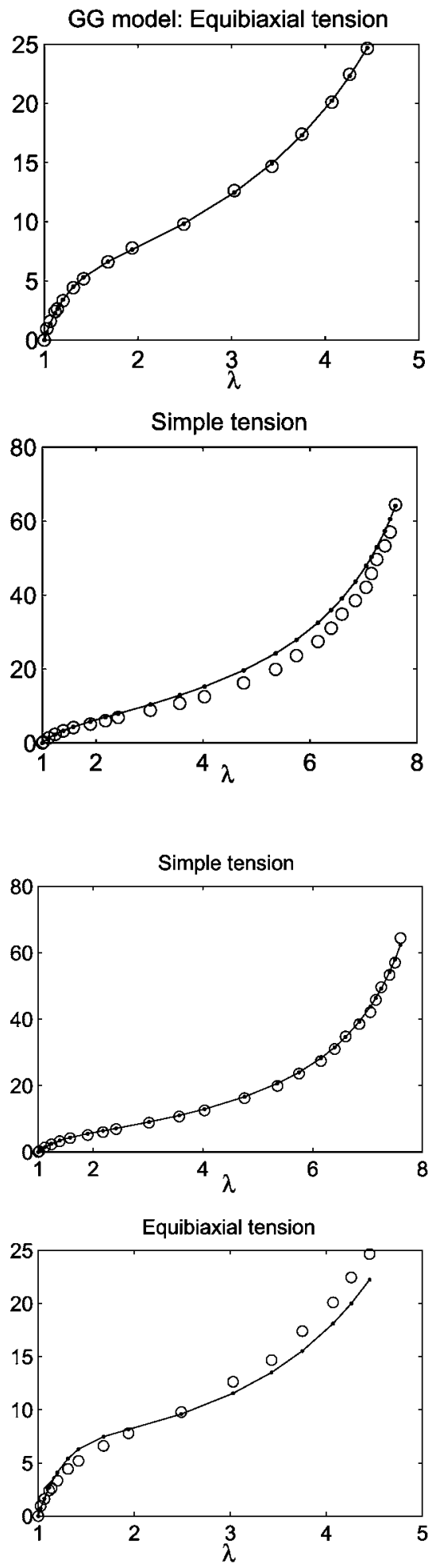
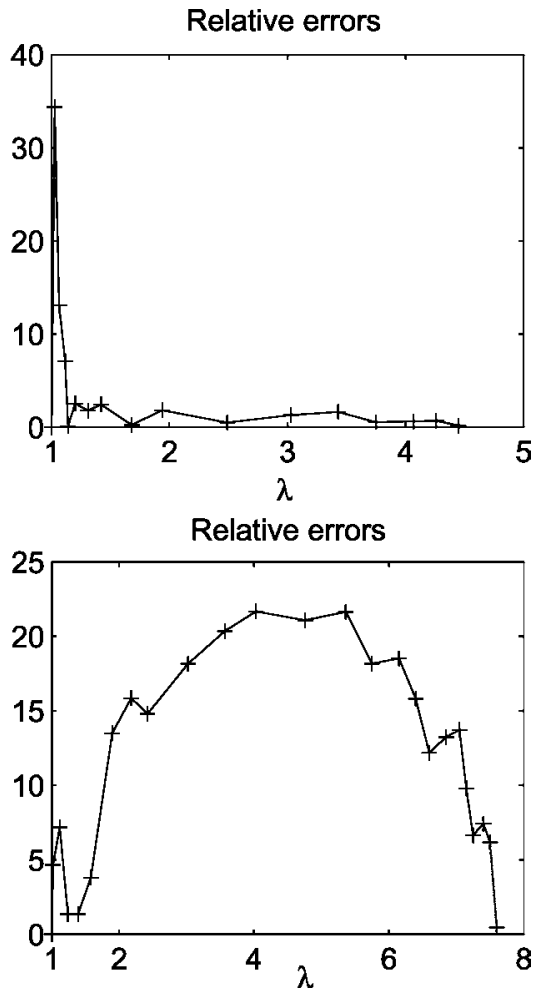

Fig. 13. Fit of the GG model to simple tension and equibiaxial tension data (circles) and the corresponding relative errors with the optimal parameter set based on use of the equibiaxial tension data. In the left-hand (right-hand) figures nominal stresses (relative errors) are plotted against stretch
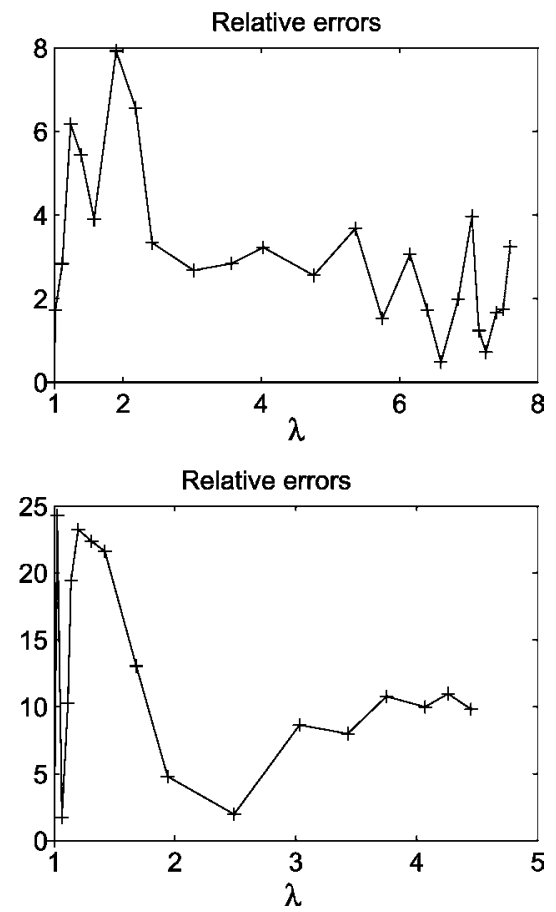

Fig. 14. Fit of the GG model to simple tension and equibiaxial tension data (circles) and the corresponding relative errors with the optimal parameter set based on use of both the simple tension and equibiaxial tension data together. In the left-hand (right-hand) figures nominal stresses (relative errors) are plotted against stretch

(simple tension). We remark that the GG model does not with residual $S=38.754, \nabla=2.58 \mathrm{e}-5, S_{\mathrm{s}}=12.574$, produce multiple local minima in the NLS optimization approximation. This is associated with the form of the log function and the fact that only three material constants are involved.

If the two data sets are fitted together according to (21) then the NLS yields the optimal set $S_{\mathrm{e}}=26.179$. The fits and the corresponding relative errors are shown in Fig. 14. Note that the coupled data do not lead to an improvement in the fit. This is because the limited number of constants does not allow enough flexi$\mu=2.5343, \quad J_{m}=79.793, \quad C_{2}=1.0368$, bility to accommodate the additional data and the constraints associated with the location of the 'limiting-chain extensibility' asymptotes. 


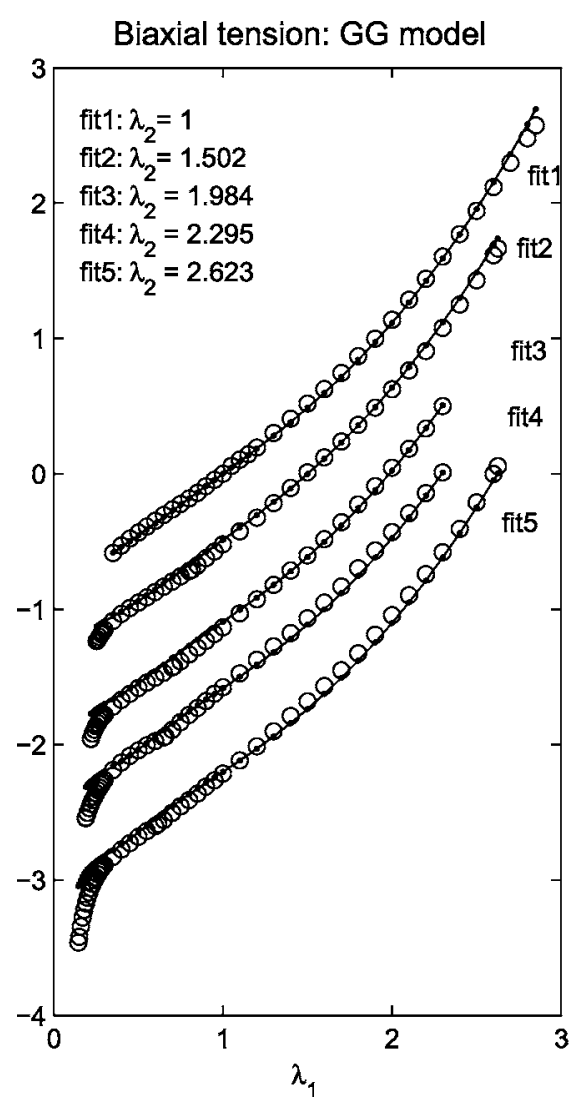

Fig. 15. Fit of the GG model to biaxial data (circles) and the relative errors. In the left-hand figure the stress difference $\sigma_{1}-\sigma_{2}$ is plotted against $\lambda_{1}$ for the fixed values of $\lambda_{2}$ indicated
If the GG model is used to fit the biaxial tension data, we find that each data set for a fixed value of $\lambda_{2}$ yields a good fit, with the values of $\mu$ and $C_{2}$ very similar in each case. For example, the set

$\mu=0.34676, \quad J_{m}=85.79, \quad C_{2}=0.05471$

is obtained for $\lambda_{2}=1$ and then used to fit the data for the other values of $\lambda_{2}$. The resulting fits are shown in Fig. 15 . The minimum and the maximum residuals with respect to all the biaxial data are $S_{\min }=0.039846, S_{\max }=0.9132$.

Note, however, that the predictive property of the biaxial tension fitting is not good for compressive strains, irrespective of the value of $J_{m}$.

Let us now apply the GG model to the biaxial case by fitting separately the data in the compression and extension ranges. Consider the data for $\lambda_{1}<1$. The solution

$\mu=0.3313, \quad J_{m}=32.823, \quad C_{2}=0.050567$

is obtained with residuals $S_{\min }=0.00045909, S_{\max }=$ 0.37933 . The fits and relative errors are shown as the upper two figures in Fig. 16. Consider next the data for $\lambda_{1} \geq 1$. Then, the solution

$\mu=0.34112, \quad J_{m}=133.52, \quad C_{2}=0.10485$

is obtained with residuals $S_{\min }=0.0010215, S_{\max }=$ 0.016084 . The corresponding fits and relative errors are shown in the lower two figures in Fig. 16.

This difference between the two estimated values of $J_{m}$ may be clarified by considering simple tension. For this case $I_{1}=\lambda^{2}+2 / \lambda$. Therefore, for fixed $J_{m}=I_{1 \max }-3$, the resulting cubic equation $\lambda^{3}-\left(J_{m}+3\right) \lambda+2=0$ for $\lambda$ has three real roots. One of these roots is negative and need not be considered; of the other roots one is less than and one greater than 1 . The roots are important because they fix the locations of the asymptotes between which the simple tension curve lies. Clearly, when the stresses for the data in tension and in compression are considered the asymptote in the compressive region and that in tension zone will not in general correspond to the same value of $J_{m}$. This suggests that the assumption of isotropy should be viewed with caution since it is clear that we do not have 'reflectional symmetry' with respect to the undeformed configuration.

To avoid this limitation of the GG model, one approach is to modify it by incorporating an element of anisotropy. A possible procedure for incorporating anisotropy in a slightly different context, but which could be adapted here, is provided by Horgan et al. [17]. In the present paper, however, we restrict attention to isotropy.

\section{6}

\section{Logarithmic strain based models}

In Criscione et al. [5], a new constitutive formulation based on certain invariants, denoted $K_{1}, K_{2}, K_{3}$, of the natural or Hencky strain is considered. The latter has principal values $\left(\ln \lambda_{1}, \ln \lambda_{2}, \ln \lambda_{3}\right)$. These invariants possess the property that they form an orthogonal invariant basis, so that the associated stress response terms are mutually orthogonal [18]. The authors assert that this is advantageous since the orthogonal entities exhibit a minimum covariance and therefore maximal independence. In particular, for incompressible materials, $K_{1} \equiv$ $\ln \left(\lambda_{1} \lambda_{2} \lambda_{3}\right)=0$, and, in terms of the stretches, the other two invariants are given as 
Biaxial tension: GG model

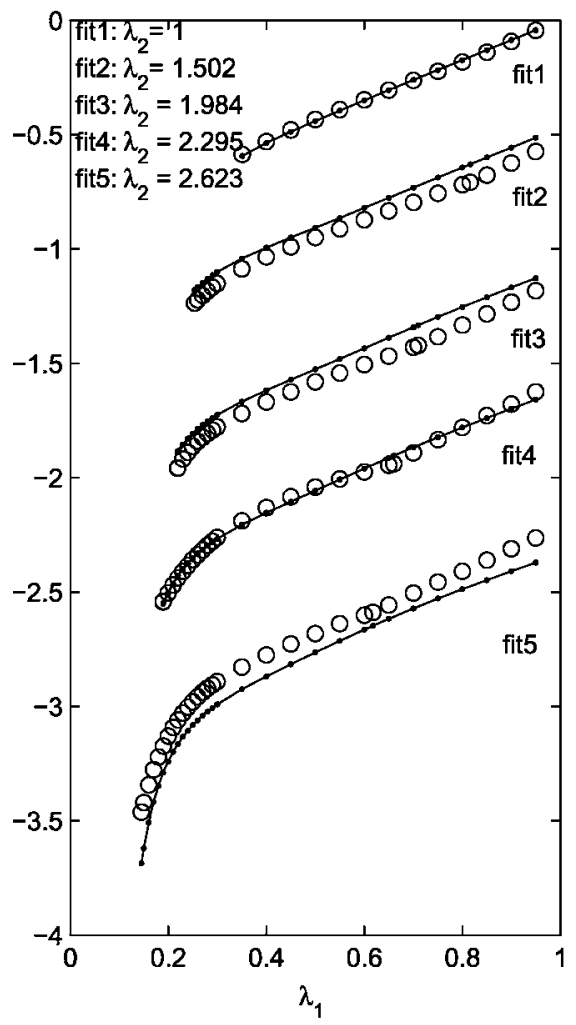

Biaxial tension: GG model

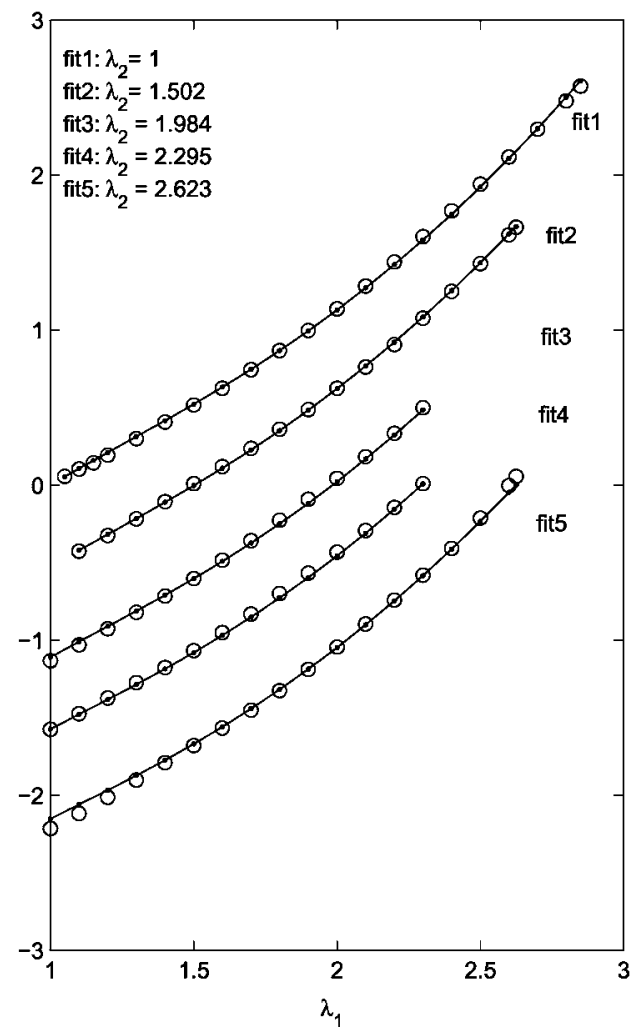

$K_{2}=\sqrt{\left(\ln \lambda_{1}\right)^{2}+\left(\ln \lambda_{2}\right)^{2}+\left(\ln \lambda_{3}\right)^{2}}$,

$K_{3}=3 \sqrt{6} \frac{\ln \lambda_{1} \ln \lambda_{2} \ln \lambda_{3}}{K_{2}^{3}}$,

\section{Relative errors}

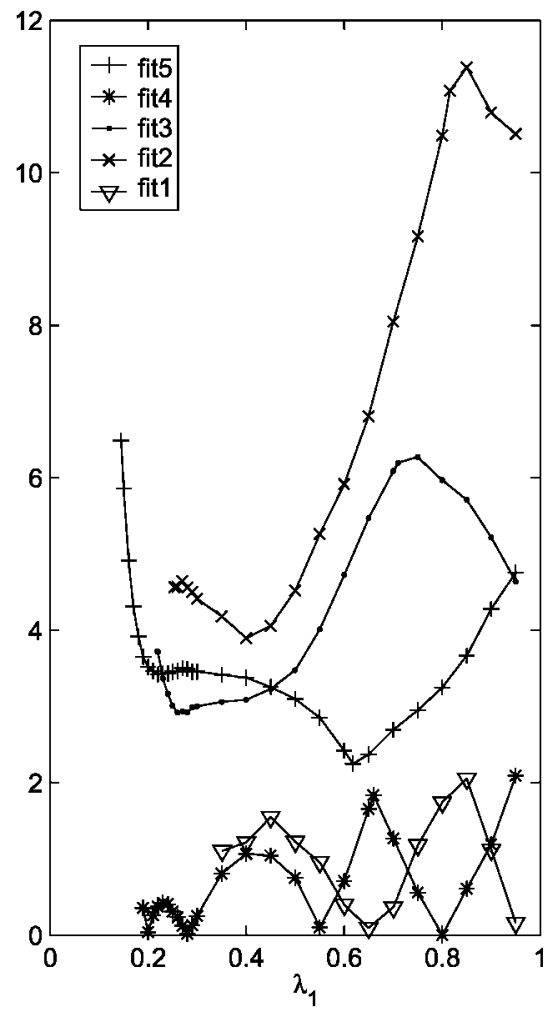

Relative errors

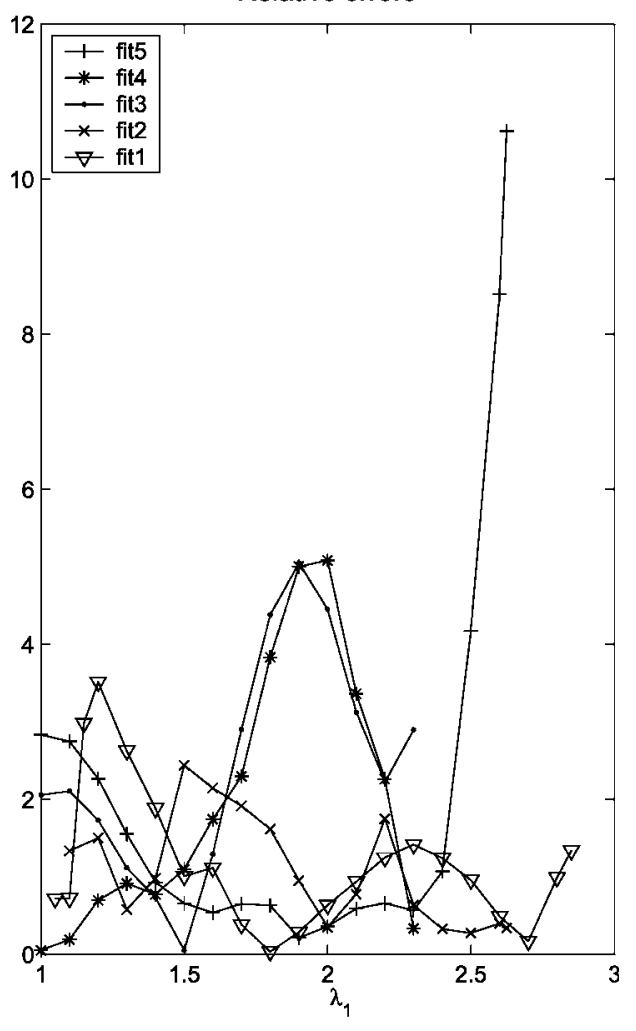

Fig. 16. Fit of the GG model and the relative errors separately for biaxial compression (upper figures) and biaxial tension (lower figures); the circles correspond to the data. In the left-hand figures the stress difference $\sigma_{1}-\sigma_{2}$ is plotted against $\lambda_{1}$ for the fixed values of $\lambda_{2}$ indicated

and the strain energy of an isotropic material may be regarded as a function of $K_{2}$ and $K_{3}: W=W\left(K_{2}, K_{3}\right)$. In [5] a specific form of energy depending linearly on $K_{3}$ is considered for purposes of comparison with the Jones-Treloar data. Explicitly, Criscione et al. [5] consider 
Simple tension: Criscione model $-\mathrm{N}=3$
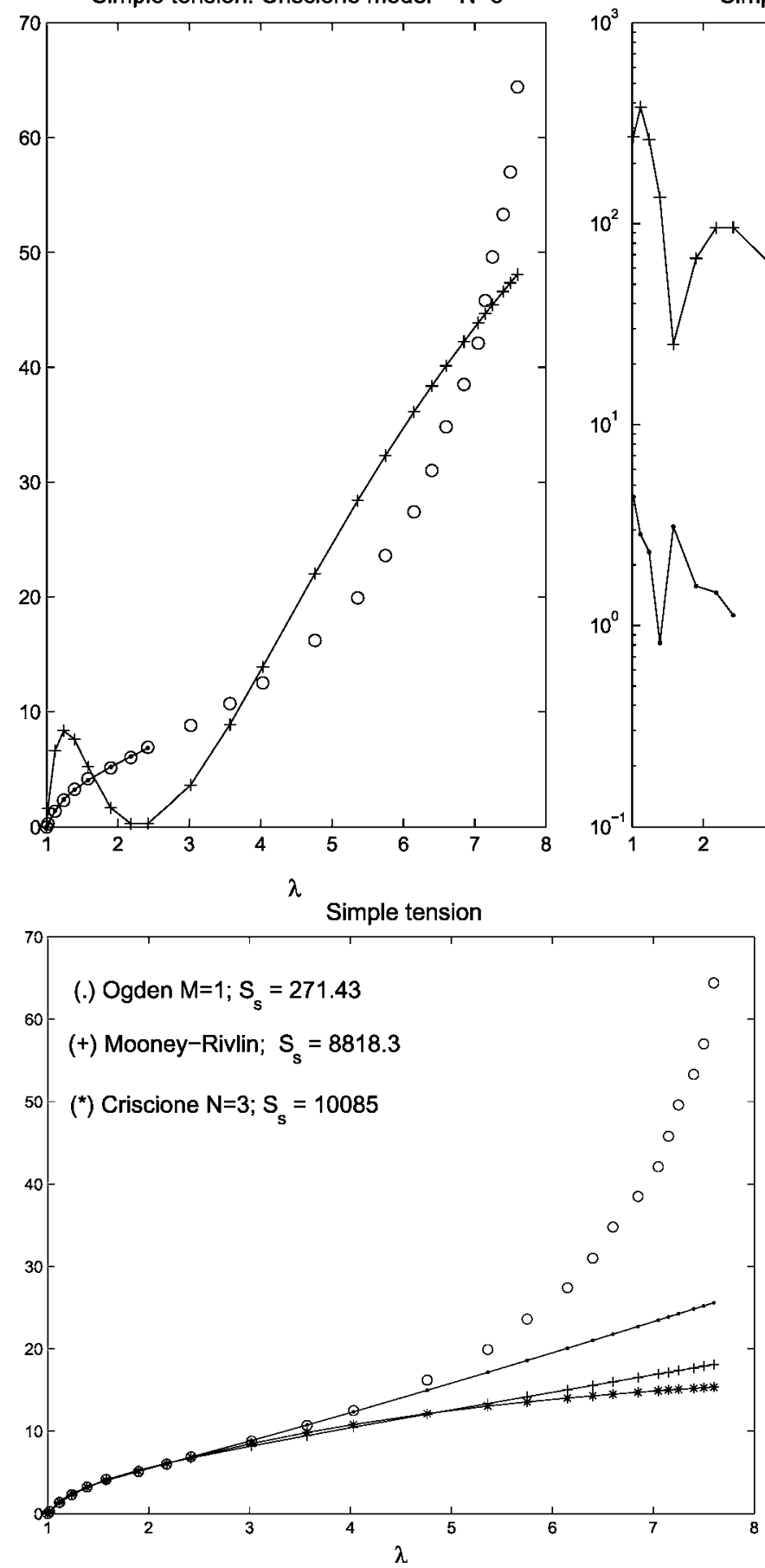

Simple tension: Relative errors

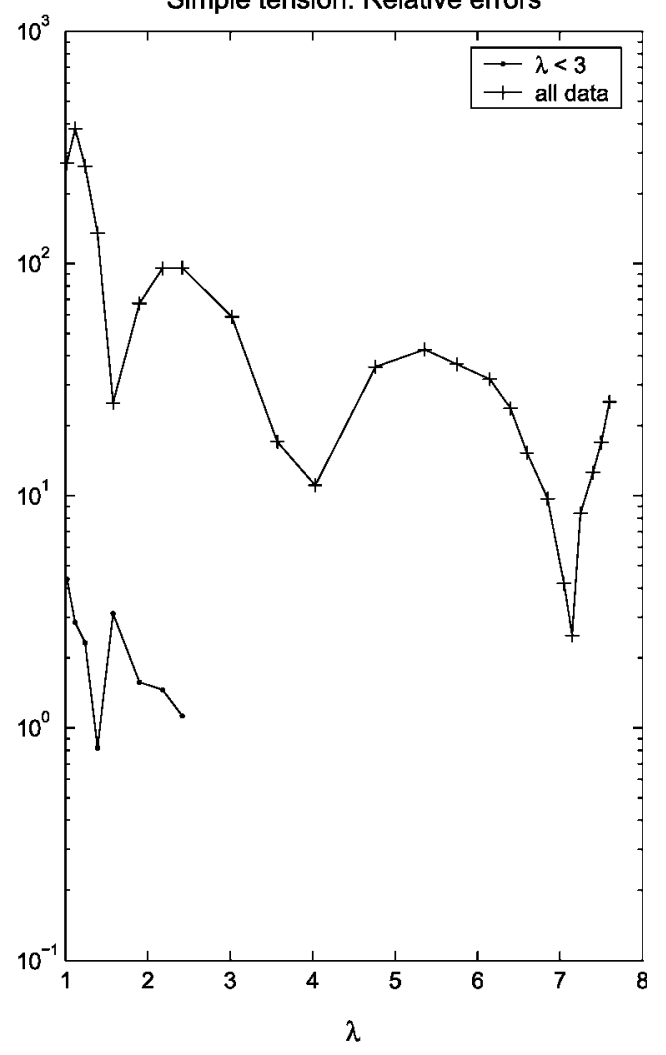

Fig. 17. Fits of the Criscione strain energy $(N=3)$ for simple tension data (circles, upper left figure) and relative errors (upper right figure), and comparison of the predictive capability on all the data (circles) with respect to the MooneyRivlin and Ogden $(M=1)$ models (lower figure)
$W\left(K_{2}, K_{3}\right)=\sum_{i=2}^{\infty} \frac{1}{i} \gamma_{i-1} K_{2}^{i}+K_{3} \sum_{j=3}^{\infty} \xi_{j} K_{2}^{j}$,

where $\gamma_{i}, i=1,2, \ldots$, and $\xi_{i}, i=3,4, \ldots$, are material constants and $\mu=\gamma_{1} / 2$ is the infinitesimal shear modulus.

The model may be approximated by a truncated $N$-term series, so that, in particular,

$\frac{\partial W}{\partial K_{2}}=\sum_{i=1}^{N} \gamma_{i} K_{2}^{i}+K_{3} \sum_{j=1}^{N-1} \xi_{j+2}(j+2) K_{2}^{j+1}$.
Then, $2 N-1$ parameters $\mathbf{p}=\left[\gamma_{1}, \ldots, \gamma_{N}, \xi_{3}, \ldots, \xi_{N+1}\right]^{\mathrm{T}}$ have to be determined.

In this case application of the LS method yields a linear problem. In fact, if $m$ is the size of the data set, an over-determined linear system $\mathrm{Ap}=\tau$ has to be solved, where $\mathbf{A}: \mathbb{R}^{(2 N-1)} \rightarrow \mathbb{R}^{m}$. In the following we denote by $S_{\mathrm{s}}=\left\|\mathbf{A}_{\mathrm{s}} \mathbf{p}-\tau_{\mathrm{s}}\right\|_{2}^{2}$ the residual for simple tension.

It is easy to show that for the simple and equibiaxial tension tests $K_{3}=1, K_{2}=\sqrt{6}(\ln \lambda) / 2$ and $K_{3}=-1$, $K_{2}=\sqrt{6} \ln \lambda$, respectively. For biaxial tension, we have 


$$
\begin{aligned}
& K_{2}=\sqrt{2} \sqrt{\left(\ln \lambda_{1}\right)^{2}+\left(\ln \lambda_{2}\right)^{2}+\ln \lambda_{1} \ln \lambda_{2}}, \\
& K_{3}=-3 \sqrt{6} \frac{\ln \lambda_{1} \ln \lambda_{2}\left(\ln \lambda_{1}+\ln \lambda_{2}\right)}{K_{2}^{3}},
\end{aligned}
$$

and the Cauchy stress difference

$\sigma_{1}-\sigma_{2}=\lambda_{1} \frac{\partial W\left(K_{2}, K_{3}\right)}{\partial \lambda_{1}}-\lambda_{2} \frac{\partial W\left(K_{2}, K_{3}\right)}{\partial \lambda_{2}}$

may be calculated accordingly.

In the analysis of the Jones-Treloar data conducted in [5] the value $N=3$ is suggested. Here, we begin the fitting of the Treloar simple tension data by taking $N=3$. We find that not only is the fit poor quantitatively, but it is also poor qualitatively and the main features of the data curve are not captured (see Fig. 17). To obtain an acceptable fitting we have determined that it is necessary to increase the value of $N$ up to 8 (which involves 15 material parameters).

If we fix $N=3$ it is possible to obtain a good fit only by restricting attention to moderate stretches $(\lambda<3$ ). (Indeed, this is the range of stretches for all the examples reported in [5].) The parameters identified for this reduced range of stretches are

$$
\begin{aligned}
\mathbf{p}_{\text {red }} & \equiv\left\{\gamma_{1}=9.7841, \gamma_{2}=-0.4506, \gamma_{3}=0.3789,\right. \\
\xi_{3} & \left.=-1.3517, \xi_{4}=1.5157\right\},
\end{aligned}
$$

with residual $S_{s}=0.0424$. The optimal set obtained for the whole range of data is

$$
\begin{aligned}
\mathbf{p}_{\text {all }} & \equiv\left\{\gamma_{1}=58.073, \gamma_{2}=-11.351, \gamma_{3}=3.2796,\right. \\
\xi_{3} & \left.=-34.053, \xi_{4}=13.118\right\},
\end{aligned}
$$

with residual $S_{s}=985.59$.

In Fig. 17 (upper) we show both fits for the Criscione model with $N=3$ and the corresponding relative errors. We note, however, that for $\lambda<3$ very good agreement is also obtained using, for example, the Ogden model for $M=1\left(\alpha_{1}=-2.1474, \mu_{1}=-1.2487, S_{\mathrm{s}}=0.0556\right)$, or the classical Mooney-Rivlin model $\left(c_{1}=1.0505, c_{2}=1.0878\right.$, $S_{\mathrm{s}}=0.0716$ ). In Fig. 17 (lower), we show the predictive capability of these two models compared with that of the $N=3$ Criscione model on the whole range of simple tension data.

In Sect. 4 and 5 we have seen that the main difference between the Ogden and GG models is that the first one admits multiple NLS solutions (for $M \geq 3$ ) while the second one admits a unique NLS solution for each data set. Concerning the linear least squares (LLS) approximation of the Criscione model, it is easy to verify that when simple, equibiaxial and biaxial tension data are considered the coefficient matrix $\mathbf{A}$ does not have full rank, which means that infinitely many solutions with the same residual are present. This is due to the choice of the particular polynomial form of $W\left(K_{2}, K_{3}\right)$. In this case, we have calculated the solution with minimum 2-norm by means of the singular value decomposition (SVD) of A according to well known formulae, given, for example, in [19].
Criscione et al. [5] did not encounter the problem of multiple solutions. In fact, they took advantage of the properties of the $K$ invariants in order to identify the parameters $\gamma=\left(\gamma_{1}, \gamma_{2}, \gamma_{3}\right)^{\mathrm{T}}$ and $\xi=\left(\xi_{3}, \xi_{4}\right)^{\mathrm{T}}$ by means of two different fits with third order $(N=3)$ polynomials, that is by solving two different LLS problems, namely

$\min \left\|\mathbf{A}_{1} \boldsymbol{\gamma}-\mathbf{b}_{1}\right\|_{2}^{2}, \quad \min \left\|\mathbf{A}_{2} \boldsymbol{\xi}-\mathbf{b}_{2}\right\|_{2}^{2}$,

where $\mathbf{A}_{1} \in \mathbb{R}^{m \times 3}, \mathbf{A}_{2} \in \mathbb{R}^{m \times 2}$ are in the class of Vandermonde matrices and $\mathbf{b}_{1}$ and $\mathbf{b}_{2}$ contain the Cauchy stress data or linear combinations of these data. In [5] these problems are solved only for pure shear $\left(\lambda_{2}=1\right)$ and the parameters identified are

$$
\begin{aligned}
& \mathbf{p}_{\mathrm{cr}} \equiv\left\{\gamma_{1}=0.96, \gamma_{2}=-0.16, \gamma_{3}=0.35, \xi_{3}=0.065\right. \\
& \left.\quad \xi_{4}=0.039\right\}
\end{aligned}
$$

The authors declare a 'good' predictive capability for this set of parameters on the simple, equibiaxial and biaxial tension data. However, we find that for simple tension the set $\mathbf{p}_{\text {cr }}$ leads to the residuals $S_{\mathrm{s}}=110.6067$ with $\lambda<3$ and $S_{\mathrm{s}}=20975$ if all the values of $\lambda$ are included. Moreover, their equibiaxial data are extracted from the biaxial ones and are different from those considered in the present paper.

It is worth noting that to obtain good fits for all the stretches it would be necessary to consider, for example, $\mathbf{A}_{1} \in \mathbb{R}^{m \times 8}$. But, it is well known that the Vandermonde matrices become ill-conditioned as the dimensions increase.

\section{7}

\section{A test boundary-value problem}

In this section we solve the boundary-value problem (BVP) arising in the modelling of rectilinear shear for the strain energies examined in Sects. 4 and 5. In particular, for the Ogden model we show how the presence of multiple solutions in the NLS approximation carries over to the approximation of the BVP solution. For this purpose, for each table in Sect. 4, we illustrate the results graphically and compare the numerical solutions corresponding to the different sets of parameters.

The differential equation and boundary conditions modelling the deformation $y(x)$ in a slab $x \in[0,1]$ are

$\Gamma\left(y^{\prime 2}\right)=\hat{\lambda}\left(x-\frac{1}{2}\right), \quad y(0)=y(1)=0$,

where

$\Gamma\left(y^{\prime 2}\right)=2\left[\bar{W}_{1}\left(y^{\prime 2}\right)+\bar{W}_{2}\left(y^{\prime 2}\right)\right]$,

$\hat{\lambda}$ is a constant and $\bar{W}_{i}\left(y^{\prime 2}\right)$ is the value of $\partial \bar{W} / \partial I_{i}$ for $I_{1}=I_{2}=3+y^{\prime 2}$. By differentiation, we transform the problem into a second-order two-point BVP and we use the function bvp4c in MATLAB 6.5 as the numerical solver. Since we are interested in comparing the solutions independently of the values of $\hat{\lambda}$, we fix it as $\hat{\lambda}=0.5$ and note that if higher values of $\hat{\lambda}$ are chosen the code is not always able to approximate the solution since boundary layers appear, in which case a different numerical method 
Table 8. Parameters for the GG strain energy

\begin{tabular}{llcl}
\hline $\mathrm{GG}$ & $\mu$ & $J_{m}$ & $C_{2}$ \\
\hline $\mathrm{GG}_{\mathrm{s}}$ & 2.4195 & 77.9310 & 1.0233 \\
$\mathrm{GG}_{\mathrm{e}}$ & 3.1674 & 88.1300 & 0.4366 \\
$\mathrm{GG}_{\text {se }}$ & 2.5343 & 79.793 & 1.0368 \\
$\mathrm{GG}_{\text {biax }}$ & 0.3468 & 85.7900 & 0.0547 \\
$\mathrm{GG}_{\text {biax-c }}$ & 0.3313 & 32.8230 & 0.0506 \\
$\mathrm{GG}_{\text {biax-e }}$ & 0.3411 & 133.5200 & 0.1049 \\
\hline
\end{tabular}

coupled with a more suitable stepsize variation strategy would be needed.

\section{1}

\section{The Ogden strain energy}

Let us solve the problem (39) using the Ogden strain energy for the cases $M=3$ and $M=4$ with the parameters reported in Tables 1 and 2 and (23) and (24) (simple tension) and in Tables 3 and 4 and (30) and (31) (equibiaxial tension). The numerical solutions are shown in Figs. 18 and 19, respectively. Included, additionally, are the results, labelled OSS ${ }^{\text {se }}$, based on the parameter sets (32) and (33) for $M=3$ and $M=4$, respectively. We note that the presence of multiple optimal sets of parameters from the NLS fittings produces numerical BVP approximations that are similar qualitatively but often very different quantitatively. In Fig. 18 the labels of the solutions corresponding to the smallest residuals in the parameter fits are shown in bold type. They are OSS 1 for $M=3$ and $\mathrm{OSS}_{3}^{s}$ for $M=4$ and we remark that in each case they corre- spond to the uppermost solution. The curves labelled set (23) and set (24) are based on the parameters in (23) and (24), respectively. In Fig. 19 the picture is similar except that the solution corresponding to the lowest residual is $\mathrm{OSS}_{1}^{\mathrm{e}}$ for both $M=3$ and $M=4$. In the case of $M=4$, however, the data set OT (from Table 4), which also has small, but a slightly larger, residual, yields the uppermost solution.

\section{2}

\section{The GG strain energy}

The BVP has also been solved for the GG strain energy for the parameters determined by the fits for all the deformations examined in Sect. 5. The optimal sets were given in Sect. 5 and are collected together here for convenience in Table 8 , in which the subscripts indicate the associated deformation (s: simple tension; e: equibiaxial tension; se: $s$ and e combined; biax: biaxial data; biax-c: biaxial data in compression; biax-e: biaxial data in extension). The corresponding solutions of the BVP are shown in Fig. 20. Note, however, that the first three sets of parameters correspond to the Treloar data and the last three to the Jones-Treloar data, which, we mention again, are for a different material.

\section{8}

\section{Concluding remarks}

In this paper we have carried out a systematic study of the fitting of stress-stretch equations for incompressible isotropic hyperelastic constitutive laws to experimental data for rubber in simple tension, equibiaxial tension and
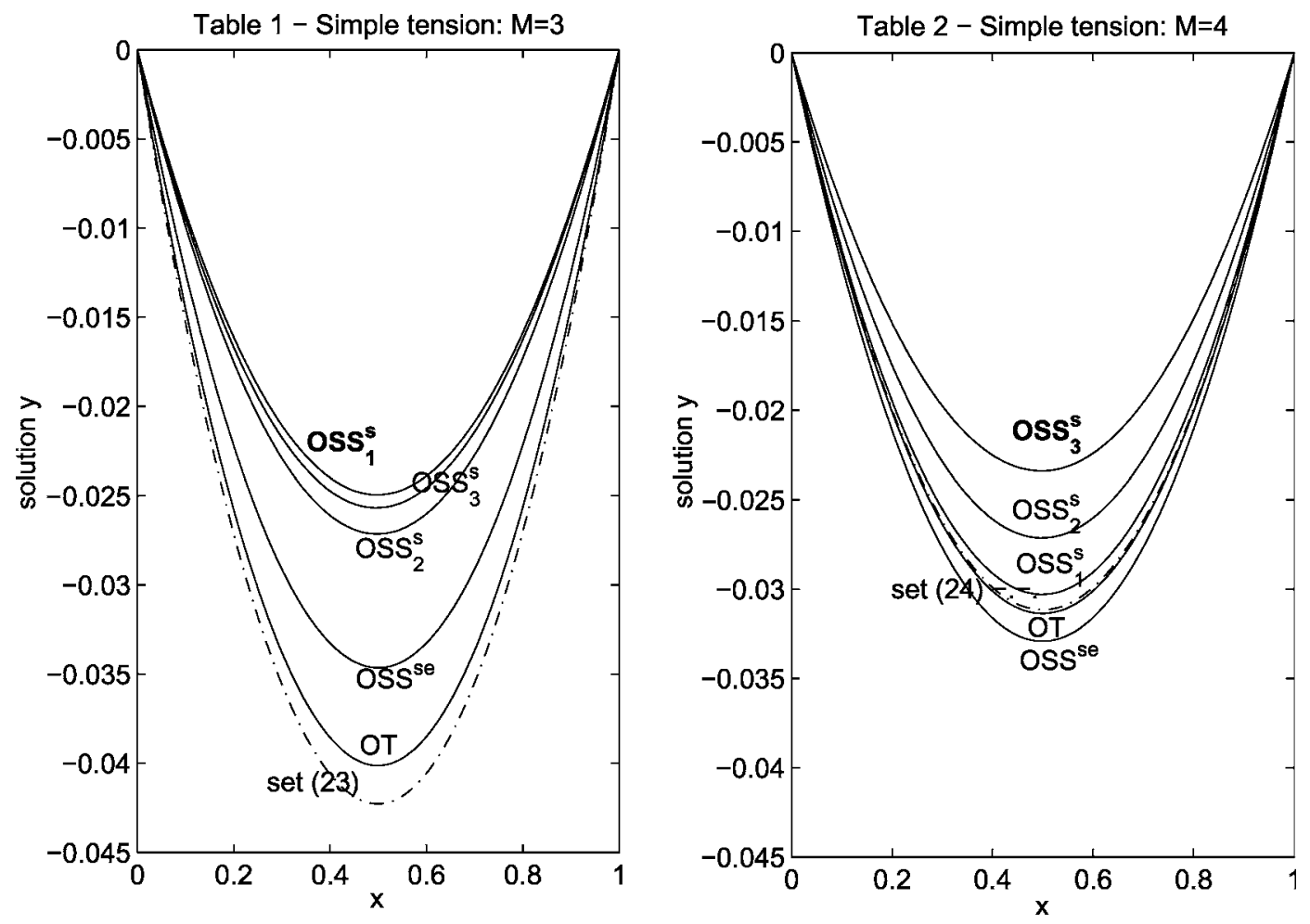

Fig. 18. Solution of the BVP (39) using the Ogden strain energy with different parameter sets obtained from the simple tension fits: $M=3$ (left figure) and $M=4$ (right figure) 


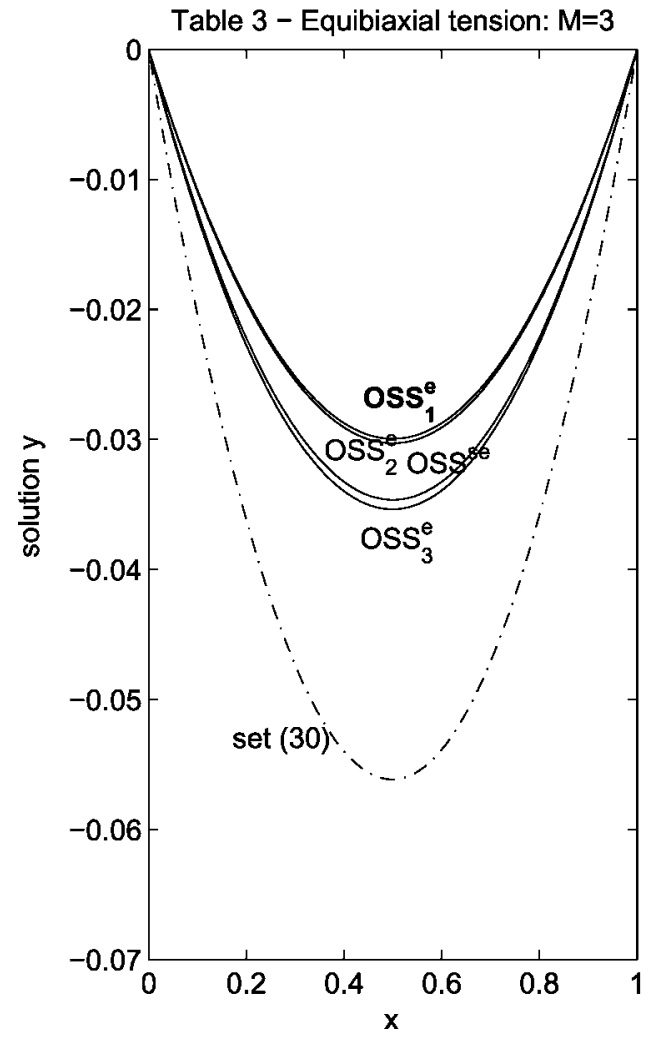

general biaxial tension tests. We have focused on the determination of material parameters and the corresponding relative errors. We have highlighted, in particular, the occurrence of multiple sets of optimal material parameters in the Ogden strain-energy function, which is formulated in terms of principal stretches, and the consequences of this for the solution of boundary-value problems. It appears that a study of this kind has not been conducted previously, although the paper by Gendy and Saleeb [20] should be mentioned since it considers certain

\section{Table 4 - Equibiaxial tension: $M=4$}

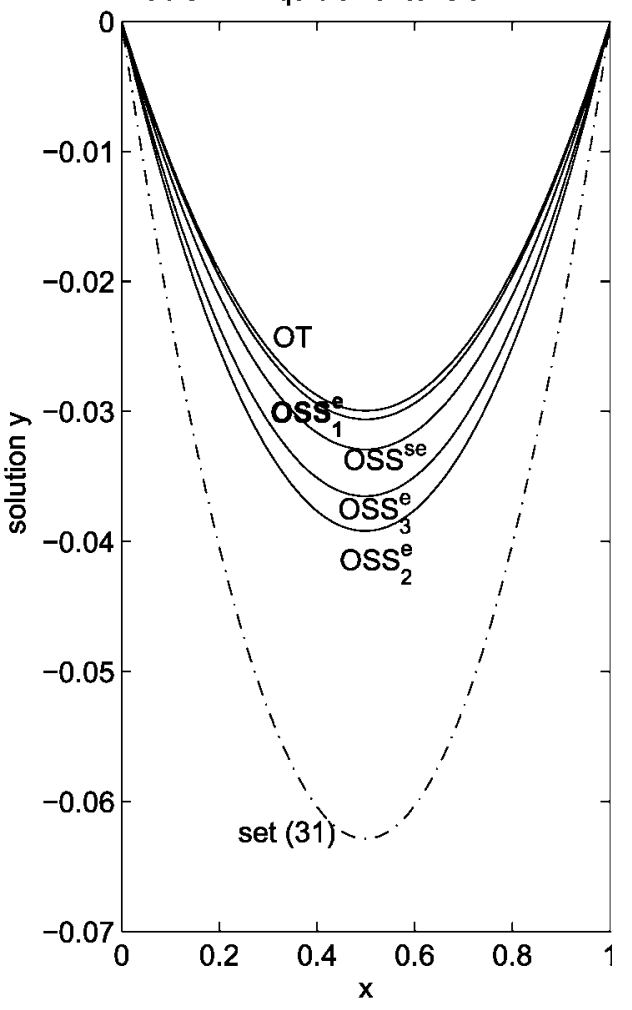

Fig. 19. Solution of the BVP (39) using the Ogden strain energy with parameters obtained from the equibiaxial tension fits: $M=3$ (left figure) and $M=4$ (right figure)

aspects of the optimization problem in respect of the Ogden strain-energy function. However, in that paper the question of multiple optimal sets of parameters was not encountered.

For comparison, we have applied a similar analysis and fitting procedure to the Pucci-Saccomandi model, which is based on the invariants $I_{1}$ and $I_{2}$, and to the Criscione model, formulated in terms of the invariants $K_{2}$ and $K_{3}$. We have found that the Pucci-Saccomandi model, considering that it involves only three material constants,

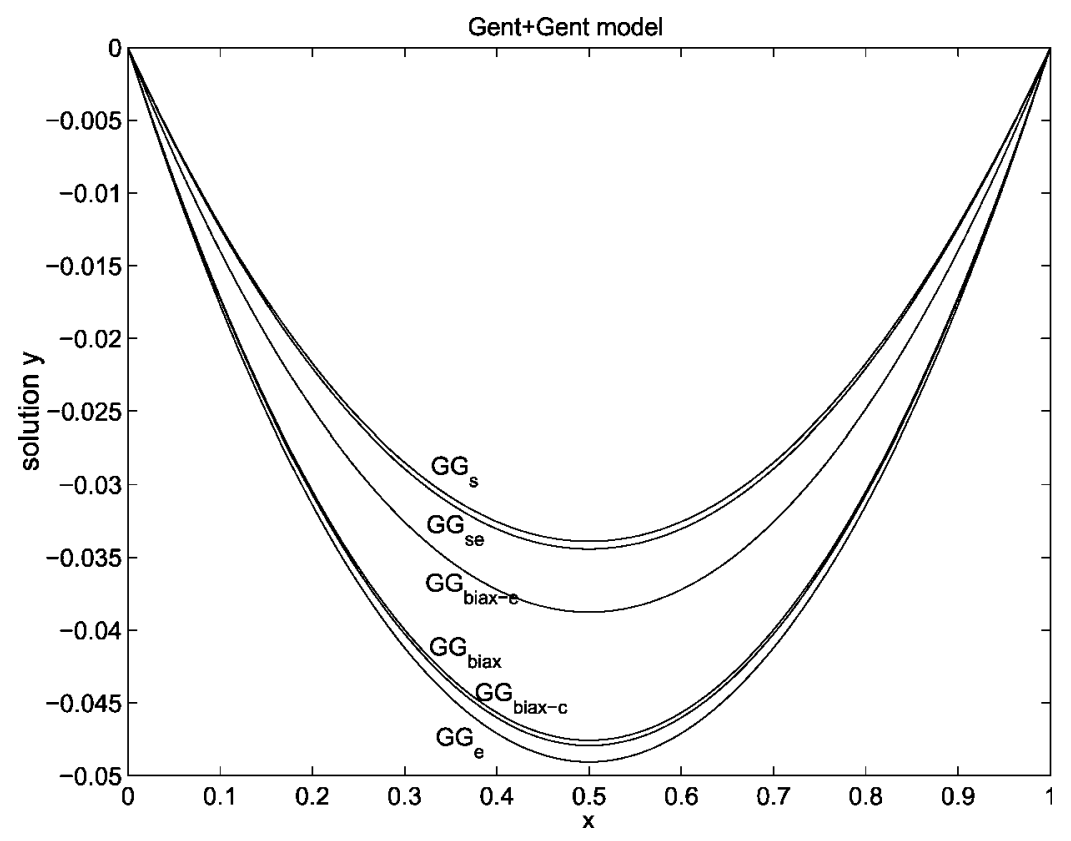

Fig. 20. Solution of the BVP (39) using the GG strain energy with parameters obtained from all the data fits with parameters given in Table 8 
gives a good fit to the range of data except for compressive values of the stretch in biaxial tests.

The Criscione model, while fitting the Jones-Treloar data well with five material constants for moderate stretches, does not provide an adequate representation of the data at larger stretches, such as those achieved in the Treloar simple tension tests, although this can be improved at the expense of introducing significantly more material parameters. Thus, the feature of the special invariants introduced in the Criscione model that they minimize the covariance between the various tensorial quantities in the Cauchy stress representation formula does not appear to be advantageous in helping to find easily and directly improved functional forms for possible strain energies. In fact, since the considered space is finite dimensional all norms are equivalent and it cannot be expected that there is a privileged set of invariants for purposes of fitting the data.

We hope that the results in this paper will be useful for improving understanding of problems encountered in fitting theory to experimental data and their practical effect on the solution of boundary-value problems. One possible outcome of this study is that, at least in the case of elastomeric materials, it is advisable to examine their behaviour first via mathematical models rather than by simulation models since the former can be designed to capture in a clear way the main qualitative features of the experimental data while the latter often aim to produce quantitative predictions by introducing large numbers of material parameters whose physical significance is unclear.

\section{References}

1. Treloar LRG (1944) Stress-strain data for vulcanized rubber under various types of deformation. Trans. Faraday Soc. 40: 59-70

2. Jones DF, Treloar LRG (1975) The properties of rubber in pure homogeneous strain. J. Phys. D, Appl. Phys. 8: 12851304

3. Ogden RW (1972) Large deformation isotropic elasticity: on the correlation of theory and experiment for incompressible rubberlike solids. Proc. R. Soc. Lond. A 326: 565-584
4. Pucci E, Saccomandi G (2002) A note on the Gent model for rubber-like materials. Rubber Chem. Technol. 75: 839-851

5. Criscione JC, Humphrey JD, Douglas AS, Hunter WC (2000) An invariant basis for natural strain which yields orthogonal stress response terms in isotropic hyperelasticity. J. Mech. Phys. Solids 48: 2445-2465

6. Ogden RW (2004) Elasticity and inelasticity of rubber. In: Saccomandi G, Ogden RW (eds) Mechanics and thermomechanics of rubberlike solids, CISM courses and lectures Series, vol. 452. Springer, Wien, pp. 135-185

7. Ogden RW (1982) Elastic deformation of rubberlike solids. In: Hopkins HG, Sewell MJ (Eds) Mechanics of Solids, The Rodney Hill 60th anniversary volume.Pergamon Press, Oxford, pp. 499-537

8. Ogden RW (1986) Recent advances in the phenomenological theory of rubber elasticity. Rubber Chem. Technol. 59: 361-383

9. Ogden RW (1997) Non-linear Elastic Deformations. Dover, New York

10. Treloar LRG (1975) The physics of rubber elasticity. Clarendon Press, Oxford

11. Beatty MF (1987) Topics in finite elasticity: hyperelasticity of rubber, elastomers, and biological tissues - with examples. Appl. Mech. Rev. 40: 1699-1733

12. Boyce MC, Arruda EM (2000) Constitutive models for rubber: a review. Rubber Chem. Technol. 73: 504-523

13. Bjorck A (1996) Numerical methods for least squares problems. SIAM Press

14. MATLAB 6.5, Release 13, Optimisation Toolbox 2.2, Users Guide

15. Twizell EH, Ogden RW (1983) Non-linear optimization of the material constants in Ogden's stress-deformation relation for incompressible isotropic elastic materials. J. Aust. Math. Soc. 24: $424-434$

16. Gent AN (1996) A new constitutive relation for rubber. Rubber Chem. Technol. 69: 59-61

17. Horgan CO, Ogden RW, Saccomandi G (2004) A theory of stress softening of elastomers based on finite chain extensibility. Proc. R. Soc. Lond. A 460: 1737-1754

18. Criscione JC (2003) Rivlin's representation formula is illconceived for the determination of response functions via biaxial testing. J. Elasticity 70: 129-147

19. Golub GH, Van Loan CF (1989) Matrix Computation. Johns Hopkins University Press, Baltimore

20. Gendy AS, Saleeb AF (2000) Nonlinear material parameter estimation for characterizing hyperelastic large strain models. Comput. Mech. 25: 66-77 